\title{
Soil Carbon Regulating Ecosystem Services in the State of South Carolina, USA
}

\author{
Elena A. Mikhailova ${ }^{1, *}$, Hamdi A. Zurqani ${ }^{1,2}{ }^{\mathbb{D}}$, Christopher J. Post ${ }^{1}$, Mark A. Schlautman ${ }^{3} \mathbb{D}$, Gregory C. Post ${ }^{4}$, \\ Lili Lin ${ }^{5}$ and Zhenbang Hao ${ }^{5}$ D \\ 1 Department of Forestry and Environmental Conservation, Clemson University, Clemson, SC 29634, USA; \\ hzurqan@clemson.edu (H.A.Z.); cpost@clemson.edu (C.J.P.) \\ 2 Department of Soil and Water Sciences, University of Tripoli, Tripoli 13538, Libya \\ 3 Department of Environmental Engineering and Earth Sciences, Clemson University, \\ Anderson, SC 29625, USA; mschlau@clemson.edu \\ 4 Economics Department, Reed College, Portland, OR 97202, USA; grpost@reed.edu \\ 5 University Key Lab for Geomatics Technology and Optimized Resources Utilization in Fujian Province, \\ No. 15 Shangxiadian Road, Fuzhou 350002, China; lili1@g.clemson.edu (L.L.); \\ zhenbanghao@fafu.edu.cn (Z.H.) \\ * Correspondence: eleanam@clemson.edu
}

Citation: Mikhailova, E.A.; Zurqani, H.A.; Post, C.J.; Schlautman, M.A.; Post, G.C.; Lin, L.; Hao, Z. Soil Carbon Regulating Ecosystem Services in the State of South Carolina, USA. Land 2021, 10, 309. https://doi.org/10.3390/land10030309

Academic Editor: Chiara Piccini

Received: 1 March 2021

Accepted: 15 March 2021

Published: 17 March 2021

Publisher's Note: MDPI stays neutral with regard to jurisdictional claims in published maps and institutional affiliations.

Copyright: (c) 2021 by the authors. Licensee MDPI, Basel, Switzerland. This article is an open access article distributed under the terms and conditions of the Creative Commons Attribution (CC BY) license (https:// creativecommons.org/licenses/by/ $4.0 /)$.

\begin{abstract}
Sustainable management of soil carbon (C) at the state level requires valuation of soil C regulating ecosystem services (ES) and disservices (ED). The objective of this study was to assess the value of regulating ES from soil organic carbon (SOC), soil inorganic carbon (SIC), and total soil carbon (TSC) stocks, based on the concept of the avoided social cost of carbon dioxide $\left(\mathrm{CO}_{2}\right)$ emissions for the state of South Carolina (SC) in the United States of America (U.S.A.) by soil order, soil depth $(0-200 \mathrm{~cm})$, region and county using information from the State Soil Geographic (STATSGO) database. The total estimated monetary mid-point value for TSC in the state of South Carolina was \$124.36B (i.e., $\$ 124.36$ billion U.S. dollars, where $\mathrm{B}=$ billion $=10^{9}$ ), $\$ 107.14 \mathrm{~B}$ for SOC, and $\$ 17.22 \mathrm{~B}$ for SIC. Soil orders with the highest midpoint value for SOC were: Ultisols (\$64.35B), Histosols (\$11.22B), and Inceptisols (\$10.31B). Soil orders with the highest midpoint value for SIC were: Inceptisols (\$5.91B), Entisols (\$5.53B), and Alfisols (\$5.0B). Soil orders with the highest midpoint value for TSC were: Ultisols (\$64.35B), Inceptisols (\$16.22B), and Entisols (\$14.65B). The regions with the highest midpoint SOC values were: Pee Dee (\$34.24B), Low Country (\$32.17B), and Midlands (\$29.24B). The regions with the highest midpoint SIC values were: Low Country (\$5.69B), Midlands (\$5.55B), and Pee Dee (\$4.67B). The regions with the highest midpoint TSC values were: Low Country (\$37.86B), Pee Dee (\$36.91B), and Midlands (\$34.79B). The counties with the highest midpoint SOC values were Colleton (\$5.44B), Horry (\$5.37B), and Berkeley (\$4.12B). The counties with the highest midpoint SIC values were Charleston $(\$ 1.46 \mathrm{~B})$, Georgetown $\left(\$ 852.81 \mathrm{M}\right.$, where $\mathrm{M}=$ million $\left.=10^{6}\right)$, and Horry $(\$ 843.18 \mathrm{M})$. The counties with the highest midpoint TSC values were Horry $(\$ 6.22 \mathrm{~B})$, Colleton $(\$ 6.02 \mathrm{~B})$, and Georgetown (\$4.87B). Administrative areas (e.g., counties, regions) combined with pedodiversity concepts can provide useful information to design cost-efficient policies to manage soil carbon regulating ES at the state level.
\end{abstract}

Keywords: accounting; carbon emissions, $\mathrm{CO}_{2}$; climate change; inorganic; organic; pedodiversity

\section{Introduction}

Economic valuation of soil carbon is vital for achieving the United Nations (UN) Sustainable Development Goals (SDGs), especially SDG 13: "Take urgent action to combat climate change and its impacts on future climate" [1]. The ecosystem services (ES) framework is often used in connection with UN SDGs because it is focused on the economic valuation of benefits (ES) and/or disservices (ED) people obtain from nature [2]. The ES framework includes three general categories of services: provisioning, regulating/maintenance, and cultural supporting services [2]. Although TSC is composed of SOC 
and SIC, only SOC is currently included in the list of soil properties important for ES [3]. Soil organic carbon is derived from living matter and tends to be concentrated in the topsoil (Table 1). In a well-aerated soil, all of the organic compounds found in plant residue are subject to enzymatic oxidation. This reaction is accompanied by oxygen consumption and $\mathrm{CO}_{2}$ release [4], which is often associated with ED in the form of realized social costs of carbon dioxide $\left(\mathrm{CO}_{2}\right)$ emissions [5]. Soil organic carbon is a fraction of soil organic matter (SOM) of $<2 \mathrm{~mm}$ particle size fraction (Table 1). Soil databases provide SOM (\%) and/or SOC (\%) in their reports listed in the tables of soil physical properties. Soil organic matter contributes to numerous soil functions (e.g., nutrient and energy reserve, etc.), which are linked to ecosystem goods and services (e.g., nutrient storage and availability, gas regulation, etc.) [6,7]. The role of SOM in delivering these ecosystem goods and services varies with scales from local (e.g., fertility maintenance) to global (e.g., mitigation of carbon emissions) [6,7]. Soil inorganic carbon, which is found in different types of carbonates (e.g., calcium, magnesium), is also essential in various ES/ED (e.g., provisioning services as a liming material for food production). It is reported as calcium carbonate $\left(\mathrm{CaCO}_{3}, \%\right)$ of $<2 \mathrm{~mm}$ particle size fraction in the tables of soil chemical properties (Table 1).

Previous research on social costs of SOC and SIC in the U.S.A. was conducted at various scales using both biophysical (e.g., soil orders) and administrative accounts (e.g., states, regions, farm, etc.) [8-10]. These analyses allowed estimation of potential social costs of soil carbon, which is useful for decision-making at the national level using detailed tables and maps of social costs of $C$ showing areas with high soil $C$ content, which can become "soil carbon hotspots" upon disturbance [10]. At the national level, the analysis showed that states have different types of soils with various soil C types (e.g., Maryland is dominated by SOC, state of New Mexico is dominated by SIC) [11], which requires soiland carbon-specific management strategies. Some states demonstrated more soil variability compared to others.

Table 1. Total soil carbon: soil organic matter (SOM), soil organic carbon (SOC), soil inorganic carbon (SIC), and carbon sequestration pathway (adapted from Mikhailova et al., 2019 [8]).

\begin{tabular}{|c|c|c|}
\hline \multicolumn{3}{|c|}{ Total soil carbon, TSC (Biotic + Abiotic) = Soil organic carbon, SOC (Biotic) + Soil inorganic carbon, SIC (Abiotic) } \\
\hline \multicolumn{2}{|c|}{ Biotic } & \multirow{2}{*}{$\begin{array}{l}\text { Abiotic } \\
\text { rganic carbon (SIC) }\end{array}$} \\
\hline $\begin{array}{l}\text { Soil organic matter (SOM) of }<2 \mathrm{~mm} \\
\text { particle size fraction }\end{array}$ & Soil organic carbon (SOC) & \\
\hline $\begin{array}{c}\text { - Fresh residue, decomposing organic } \\
\text { matter, stable organic matter (humus), } \\
\text { and living organisms. } \\
\text { or } \\
\text { - "Continuum of organic material in all } \\
\text { stages of transformation and } \\
\text { decomposition or stabilization [12]." }\end{array}$ & $\begin{array}{l}\text { - Carbon fraction of soil organic matter of } \\
\quad<2 \mathrm{~mm} \text { particle size fraction. }\end{array}$ & $\begin{array}{l}\text { - Carbon fraction of calcium carbonate } \\
\left(\mathrm{CaCO}_{3}\right) \text { of }<2 \mathrm{~mm} \text { particle size fraction. }\end{array}$ \\
\hline \multicolumn{2}{|c|}{$\begin{array}{c}\text { Conversion (using Van Bemmelen factor of } 0.58 \text { or 1.724): } \\
\text { SOM }(\%)=\operatorname{SOC}(\%) \times 1.724 \text { or } \\
\text { SOC }(\%)=\operatorname{SOM}(\%) \times 0.58[13]\end{array}$} & $\begin{array}{l}\text { Conversion: } \mathrm{CaCO}_{3}(\%)=\mathrm{SIC}(\%) \times \\
100 / 12 \text { or SIC }(\%)=\mathrm{CaCO}_{3}(\%) \times 0.12\end{array}$ \\
\hline $\begin{array}{r}\text { Pathways to increased C sequestration: } \\
\text { additions, etc.); land/agricultural ma } \\
\text { conservation, etc.); a }\end{array}$ & $\begin{array}{l}\text { Additions of organic matter (e.g., compost } \\
\text { hagement (e.g., no-till operations, land } \\
\text { forestation, etc. }[6,7] \text {. }\end{array}$ & $\begin{array}{l}\text { Pathways to increased } \mathrm{C} \text { sequestration: } \\
\text { Additions of } \mathrm{Ca}^{2+} \text { and } \mathrm{Mg}^{2+} \text { cations } \\
\text { outside the soil (e.g., atmospheric } \\
\text { deposition, etc.) [14]. }\end{array}$ \\
\hline
\end{tabular}




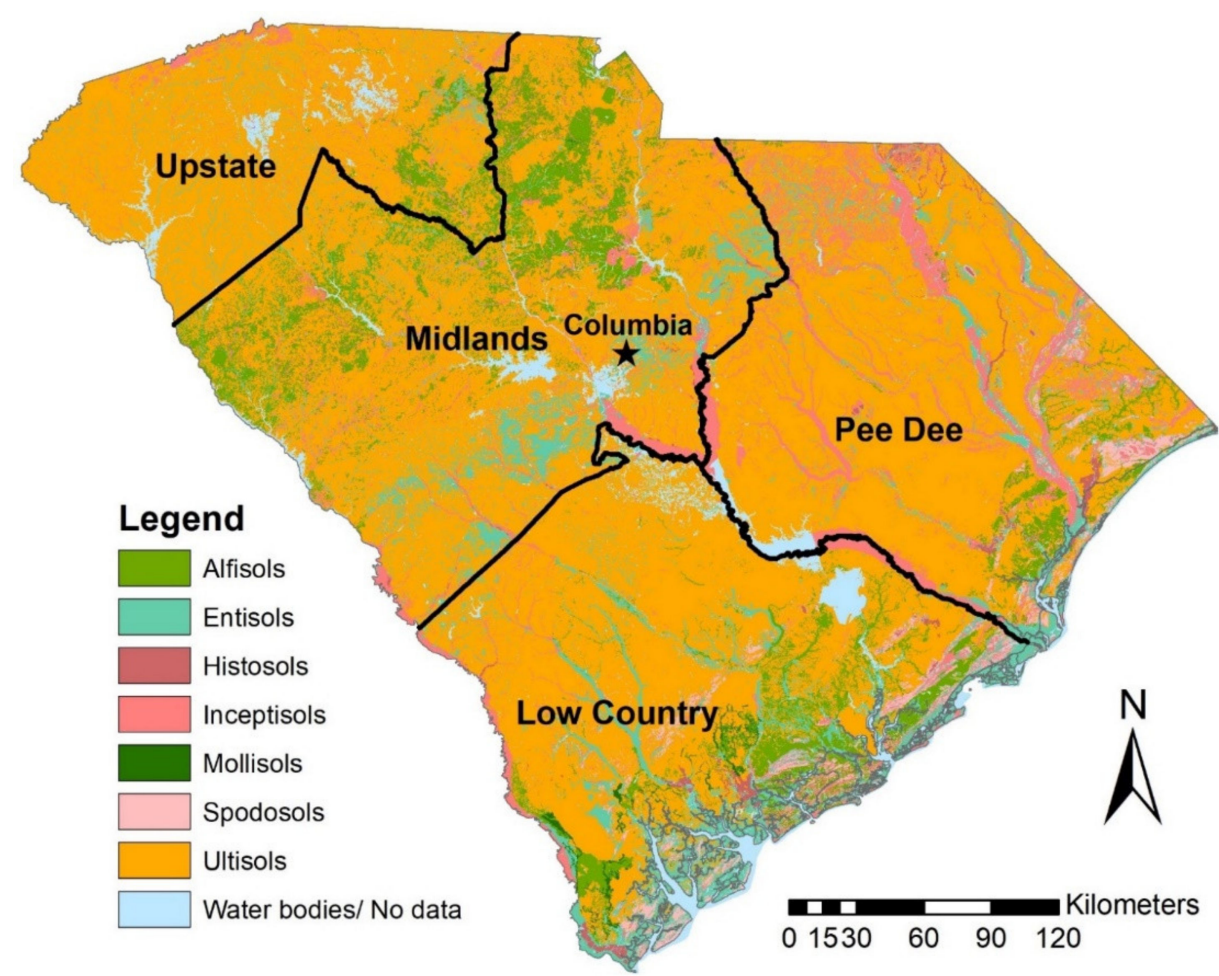

Figure 1. General soil map of South Carolina (U.S.A.) $\left(33.8361^{\circ} \mathrm{N}, 81.1637^{\circ} \mathrm{W}\right)$ (adapted from [15]).

The ES framework is increasingly being used to address environmental concerns (e.g., global warming, climate change, etc.), but because of "the difficulty in relating soil properties to ES, soil ES are still not fully considered in the territorial planning decision process" [16]. According to Fossey et al., 2020 [16], soil databases play an essential role in assessing ES/ED in territorial planning. For sustainable soil $\mathrm{C}$ management decisions at the state level and its counties, it is critical to determine soil $C$ and the distribution of its social costs within the state overall and by individual counties linked to biophysical units (e.g., soil orders). This type of analysis will allow prioritization of soil $\mathrm{C}$ management within the state based on this distribution. The hypothesis of this study is that pedodiversity concepts overlayed with administrative units (Figures 1 and 2) can be used to identify spatial patterns of soil carbon hotspots for sustainable management.

The specific objective of this study was to assess the value of SOC, SIC, and TSC in the state of South Carolina (U.S.A.) based on the social cost of carbon $\left(\mathrm{SC}-\mathrm{CO}_{2}\right)$ and avoided emissions provided by carbon sequestration, which the U.S. Environmental Protection Agency (EPA) has determined to be $\$ 46$ per metric ton of $\mathrm{CO}_{2}$, which is applicable for the year 2025 based on 2007 U.S. dollars and an average discount rate of 3\% [17]. This study provides the monetary values of SOC, SIC, and TSC for soil depth $(0-200 \mathrm{~cm})$ across the state and by considering different spatial aggregation levels (i.e., region, county) using State Soil Geographic (STATSGO) database, and information previously reported by Guo et al. (2006) [18].

\section{Materials and Methods}

The Accounting Framework

This study used both biophysical (science-based, Figure 1) and administrative (boundarybased, Figure 3) accounts to calculate monetary values for SOC, SIC, and TSC (Tables 2 and 3). 
Table 2. A conceptual overview of the accounting framework used in this study (adapted from Groshans et al., 2018 [19]).

\begin{tabular}{ccccc}
\hline $\begin{array}{c}\text { Biophysical Accounts } \\
\text { (Science-Based) }\end{array}$ & $\begin{array}{c}\text { Administrative Accounts } \\
\text { (Boundary-Based) }\end{array}$ & Monetary Account(s) & Benefit(s) & Total Value \\
\hline Soil extent: & Administrative extent: & $\begin{array}{c}\text { Ecosystem good(s) and } \\
\text { service(s): }\end{array}$ & Sector: & Types of value: \\
\hline Separate constitute stock 1: Soil organic carbon (SOC) & Separate constitute stock 2: Soil inorganic carbon (SIC) \\
\hline Composite (total) stock: Total soil carbon (TSC) = Soil organic carbon (SOC) + Soil inorganic carbon (SIC)
\end{tabular}

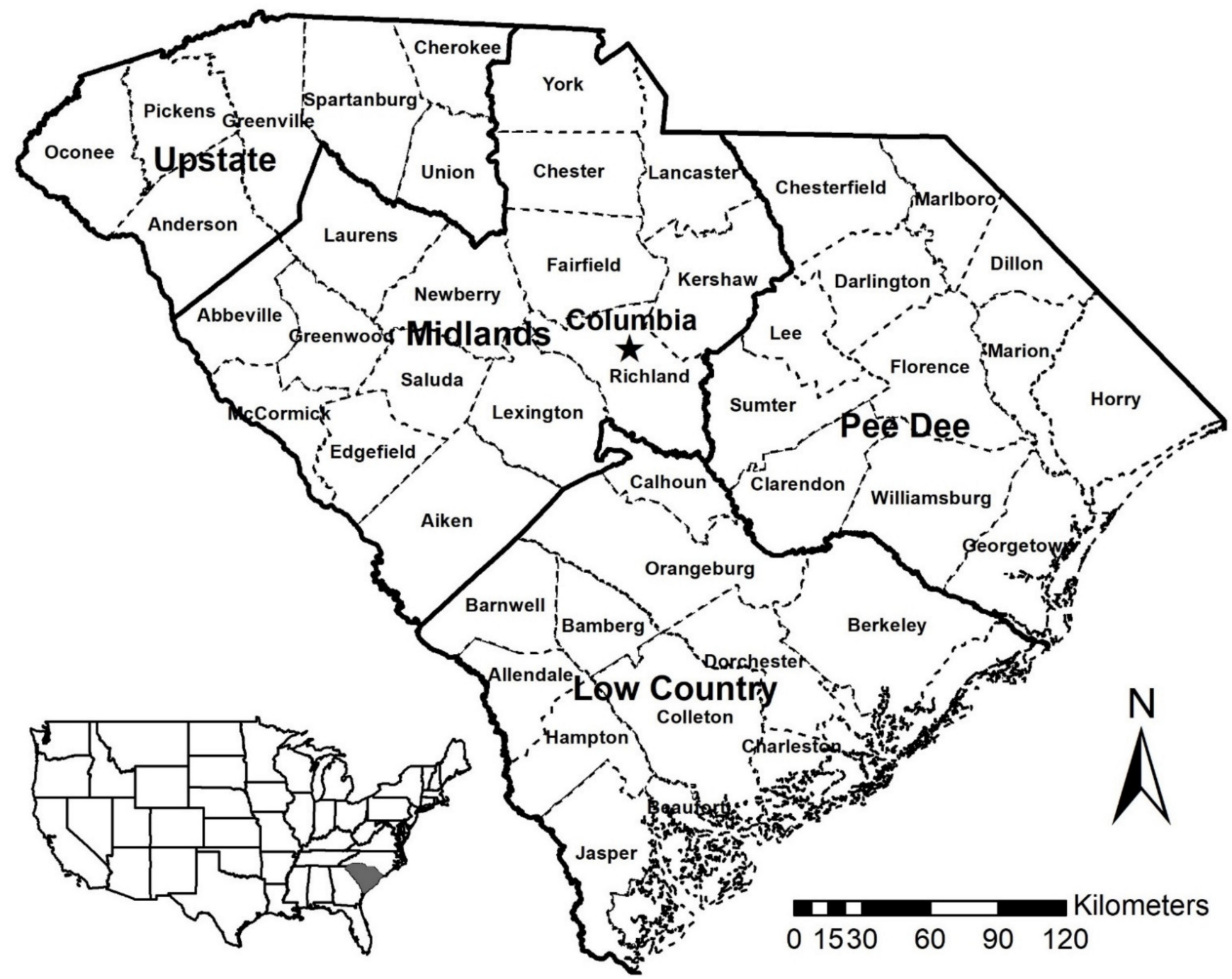

Figure 2. Administrative map of South Carolina (U.S.A.) $\left(33.8361^{\circ} \mathrm{N}, 81.1637^{\circ} \mathrm{W}\right)$ with 46 counties and four regions [20]. 
Table 3. Soil diversity (pedodiversity) by soil order (taxonomic pedodiversity), region, and county in South Carolina (U.S.A.) based on Soil Survey Geographic (SSURGO) Database (2020) [15].

\begin{tabular}{|c|c|c|c|c|c|c|c|c|}
\hline \multirow{4}{*}{ County (Region) } & \multirow{4}{*}{$\begin{array}{c}\text { Total Area } \\
\left(\mathbf{k m}^{2}\right) \\
(\text { Rank) }\end{array}$} & \multicolumn{7}{|c|}{ Slight $<\longrightarrow$ Degree of Weathering and Soil Development $\longrightarrow$ Strong } \\
\hline & & \multicolumn{3}{|c|}{ Slightly Weathered } & \multicolumn{2}{|c|}{ Moderately Weathered } & \multicolumn{2}{|c|}{ Strongly Weathered } \\
\hline & & Entisols & Inceptisols & Histosols & Alfisols & Mollisols & Spodosols & Ultisols \\
\hline & & \multicolumn{7}{|c|}{ Area $\left(\mathrm{km}^{2}\right)$} \\
\hline Anderson & $1841(14)$ & 102 & 0 & 0 & 0 & 0 & 0 & 1739 \\
\hline Cherokee & $1012(44)$ & 118 & 94 & 0 & 100 & 0 & 0 & 700 \\
\hline Greenville & $1916(11)$ & 108 & 166 & 0 & 0 & 0 & 0 & 1642 \\
\hline Oconee & $1620(23)$ & 37 & 56 & 0 & 0 & 0 & 0 & 1527 \\
\hline Pickens & $1257(35)$ & 39 & 55 & 0 & 0 & 0 & 0 & 1163 \\
\hline Spartanburg & $1881(12)$ & 8 & 161 & 0 & 77 & 0 & 0 & 1635 \\
\hline Union & $1322(32)$ & 74 & 61 & 0 & 364 & 0 & 0 & 823 \\
\hline (Upstate) & 10,849 (4) & 486 & 593 & 0 & 541 & 0 & 0 & 9229 \\
\hline Abbeville & $1269(34)$ & 52 & 38 & 0 & 401 & 0 & 0 & 778 \\
\hline Aiken & $2758(4)$ & 445 & 213 & 11 & 5 & 0 & 0 & 2084 \\
\hline Chester & $1493(25)$ & 60 & 82 & 0 & 570 & 6 & 0 & 775 \\
\hline Edgefield & $1289(33)$ & 101 & 56 & 0 & 61 & 0 & 0 & 1071 \\
\hline Fairfield & $1683(20)$ & 1 & 175 & 0 & 585 & 0 & 0 & 922 \\
\hline Greenwood & $1171(38)$ & 0 & 116 & 0 & 335 & 0 & 0 & 720 \\
\hline Kershaw & $1871(13)$ & 383 & 161 & 20 & 47 & 0 & 0 & 1260 \\
\hline Lancaster & $1409(30)$ & 53 & 151 & 0 & 62 & 0 & 0 & 1143 \\
\hline Laurens & 1837 (15) & 125 & 19 & 0 & 351 & 0 & 0 & 1342 \\
\hline Lexington & 1756 (17) & 454 & 86 & 0 & 36 & 0 & 10 & 1170 \\
\hline Newberry & $1621(22)$ & 70 & 64 & 0 & 278 & 0 & 0 & 1209 \\
\hline Richland & $1827(16)$ & 163 & 360 & 8 & 19 & 0 & 0 & 1277 \\
\hline Saluda & 1170 (39) & 19 & 83 & 0 & 77 & 0 & 0 & 991 \\
\hline York & $1753(18)$ & 5 & 134 & 0 & 577 & 0 & 0 & 1037 \\
\hline (Midlands) & 22,899 (1) & 1931 & 1738 & 31 & 3404 & 6 & 10 & 15,779 \\
\hline Chesterfield & $2053(9)$ & 173 & 655 & 0 & 23 & 0 & 0 & 1202 \\
\hline Clarendon & $1566(24)$ & 39 & 192 & 6 & 0 & 0 & 0 & 1329 \\
\hline Darlington & $1442(28)$ & 36 & 258 & 9 & 0 & 0 & 1 & 1138 \\
\hline Dillon & 1040 (42) & 91 & 128 & 8 & 0 & 0 & 19 & 794 \\
\hline Florence & $2046(10)$ & 97 & 224 & 0 & 0 & 0 & 2 & 1723 \\
\hline Georgetown & $2064(8)$ & 351 & 274 & 57 & 409 & 0 & 115 & 858 \\
\hline Horry & $2888(1)$ & 252 & 431 & 64 & 287 & 0 & 330 & 1524 \\
\hline Lee & $1058(40)$ & 29 & 131 & 0 & 1 & 0 & 0 & 897 \\
\hline Marion & 1241 (36) & 107 & 286 & 27 & 0 & 0 & 49 & 772 \\
\hline Marlboro & $1230(37)$ & 75 & 269 & 81 & 17 & 0 & 2 & 786 \\
\hline Sumter & 1694 (19) & 9 & 350 & 0 & 4 & 0 & 2 & 1329 \\
\hline Williamsburg & $2400(6)$ & 25 & 209 & 0 & 0 & 0 & 3 & 2163 \\
\hline (Pee Dee) & $20,722(3)$ & 1284 & 3407 & 252 & 741 & 0 & 523 & 14,515 \\
\hline Allendale & 1055 (41) & 25 & 101 & 6 & 0 & 0 & 0 & 923 \\
\hline Bamberg & $1018(44)$ & 126 & 1 & 0 & 41 & 0 & 3 & 847 \\
\hline Barnwell & $1416(29)$ & 78 & 138 & 0 & 0 & 0 & 0 & 1200 \\
\hline Beaufort & $1402(31)$ & 698 & 34 & 6 & 40 & 23 & 210 & 391 \\
\hline Berkeley & $2809(3)$ & 145 & 208 & 23 & 409 & 0 & 137 & 1887 \\
\hline Calhoun & $748(46)$ & 66 & 26 & 0 & 0 & 0 & 0 & 656 \\
\hline Charleston & $2317(7)$ & 765 & 332 & 0 & 727 & 0 & 273 & 220 \\
\hline Colleton & $2677(5)$ & 280 & 49 & 88 & 140 & 85 & 109 & 1,926 \\
\hline Dorchester & $1455(26)$ & 274 & 17 & 1 & 280 & 0 & 30 & 853 \\
\hline Hampton & $1443(27)$ & 136 & 87 & 3 & 101 & 0 & 40 & 1076 \\
\hline Jasper & $1669(21)$ & 318 & 58 & 57 & 246 & 116 & 24 & 850 \\
\hline McCormick & $921(45)$ & 64 & 65 & 0 & 247 & 0 & 0 & 545 \\
\hline Orangeburg & $2844(2)$ & 73 & 18 & 0 & 25 & 0 & 1 & 2,727 \\
\hline (Low Country) & 21,774 (2) & 3048 & 1134 & 184 & 2256 & 224 & 827 & 14,101 \\
\hline Totals & 76,252 & 6749 & 6872 & 475 & 6942 & 230 & 1360 & 53,624 \\
\hline
\end{tabular}


The present study is based on the SOC [21], SIC [21], TSC estimated values for the SOC, SIC, and TSC storage (in $\mathrm{Mg}$ or metric tons) and content (in $\mathrm{kg} \mathrm{m}^{-2}$ ) in the contiguous U.S. from Guo et al. (2006) [18]. A monetary valuation for TSC was calculated using the social cost of carbon $\left(\mathrm{SC}-\mathrm{CO}_{2}\right)$ of $\$ 46$ per metric ton of $\mathrm{CO}_{2}$, which is applicable for 2025 based on 2007 U.S. dollars and an average discount rate of 3\% [17]. According to the EPA, the $\mathrm{SC}-\mathrm{CO}_{2}$ is intended to be a comprehensive estimate of climate change damages. Still, it can underestimate the true damages and cost of $\mathrm{CO}_{2}$ emissions due to the exclusion of various important climate change impacts recognized in the literature [17]. Soil carbon (SC) storage and content numbers were then converted to U.S. dollars and dollars per square meter in Microsoft Excel using the following equations, with a social cost of carbon of $\$ 46 / \mathrm{Mg} \mathrm{CO}_{2}$ :

$$
\begin{gathered}
\$=(\text { SC Storage, } \mathrm{Mg}) \times \frac{44 \mathrm{MgCO}}{12 \mathrm{Mg} \mathrm{TSC}} \times \frac{\$ 46}{\mathrm{MgCO}_{2}} \\
\frac{\$}{\mathrm{~m}^{2}}=\left(\text { SC Content }, \frac{\mathrm{kg}}{\mathrm{m}^{2}}\right) \times \frac{1 \mathrm{Mg}}{10^{3} \mathrm{~kg}} \times \frac{44 \mathrm{MgCO}}{12 \mathrm{Mg} \mathrm{TSC}} \times \frac{\$ 46}{\mathrm{MgCO}}
\end{gathered}
$$

Table 4 presents area-normalized content $\left(\mathrm{kg} \mathrm{m}^{-2}\right)$ and monetary values $\left(\$ \mathrm{~m}^{-2}\right)$ of soil carbon, which were used to estimate total soil carbon storage and total soil carbon value by multiplying corresponding content (values) numbers by an area of a particular soil order within a county (region) (Table 3). For example, for the soil order of Entisols, Guo et al. (2006) [18] reported an area-normalized midpoint SOC content number of $8.0 \mathrm{~kg} \cdot \mathrm{m}^{-2}$ in the upper $2 \mathrm{~m}$ (Table 4), which was used to calculate the total SOC storage in soil order by multiplying its area in particular county or region. Then, the reported area-normalized midpoint SOC content number of $8.0 \mathrm{~kg} \cdot \mathrm{m}^{-2}$ in the upper $2 \mathrm{~m}$ (Table 4) was converted to monetary values $\left(\$ \mathrm{~m}^{-2}\right)$ of soil organic carbon using a social cost of carbon $\left(\mathrm{SC}-\mathrm{CO}_{2}\right)$ of $\$ 46$ per metric ton of $\mathrm{CO}_{2}$ (2007 U.S. dollars with an average discount rate of 3\% [17]), which is $\$ 1.35 \mathrm{~m}^{-2}$ to calculate the total monetary value of SOC storage.

Table 4. Area-normalized content $\left(\mathrm{kg} \mathrm{m}^{-2}\right)$ and monetary values $\left(\$ \mathrm{~m}^{-2}\right)$ of soil organic carbon (SOC), soil inorganic carbon (SIC), total soil carbon (TSC) by soil order based on numbers in the upper $2 \mathrm{~m}$ of the soil based on data from Guo et al., 2006 [18]

\begin{tabular}{|c|c|c|c|c|c|c|}
\hline \multirow{3}{*}{ Soil Order } & SOC Content & SIC Content & TSC Content & SOC Value & SIC Value & TSC Value \\
\hline & \multicolumn{3}{|c|}{ Minimum-Midpoint-Maximum Values } & \multicolumn{3}{|c|}{ Midpoint Values } \\
\hline & $\left(\mathrm{kg} \mathrm{m}^{-2}\right)$ & $\left(\mathrm{kg} \mathrm{m}^{-2}\right)$ & $\left(\mathrm{kg} \mathrm{m}^{-2}\right)$ & $\left(\$ m^{-2}\right)$ & $\left(\$ \mathrm{~m}^{-2}\right)$ & $\left(\$ m^{-2}\right)$ \\
\hline \multicolumn{7}{|c|}{ Slightly Weathered } \\
\hline Entisols & $1.8-8.0-15.8$ & $1.9-4.8-8.4$ & $3.7-12.8-24.2$ & 1.35 & 0.82 & 2.17 \\
\hline Inceptisols & 2.8-8.9-17.4 & $2.5-5.1-8.4$ & 5.3-14.0-25.8 & 1.50 & 0.86 & 2.36 \\
\hline Histosols & $63.9-140.1-243.9$ & $0.6-2.4-5.0$ & $64.5-142.5-248.9$ & 23.62 & 0.41 & 24.03 \\
\hline \multicolumn{7}{|c|}{ Moderately Weathered } \\
\hline Alfisols & $2.3-7.5-14.1$ & $1.3-4.3-8.1$ & $3.6-11.8-22.2$ & 1.27 & 0.72 & 1.99 \\
\hline Mollisols & $5.9-13.5-22.8$ & $4.9-11.5-19.7$ & $10.8-25.0-42.5$ & 2.28 & 1.93 & 4.21 \\
\hline \multicolumn{7}{|c|}{ Strongly Weathered } \\
\hline Spodosols & 2.9-12.3-25.5 & $0.2-0.6-1.1$ & $3.1-12.9-26.6$ & 2.07 & 0.10 & 2.17 \\
\hline Ultisols & 1.9-7.1-13.9 & $0.0-0.0-0.0$ & 1.9-7.1-13.9 & 1.20 & 0.00 & 1.20 \\
\hline
\end{tabular}
and a social cost of carbon (SC- $\mathrm{CO}_{2}$ ) of $\$ 46$ per metric ton of $\mathrm{CO}_{2}$ (2007 U.S. dollars with an average discount rate of 3\% [17]).

Note: TSC $=$ SOC + SIC. 


\section{Results}

The total estimated monetary mid-point value for TSC in the state of South Carolina was $\$ 124.36 \mathrm{~B}$ (i.e., $\$ 124.36$ billion U.S. dollars, where $\mathrm{B}=$ billion $=10^{9}$ ), $\$ 107.14 \mathrm{~B}$ for SOC, and \$17.22B for SIC. The state of South Carolina ranked 31st for TSC, 25th for SOC, and 32nd for SIC. Figure 3 shows the distribution of soil carbon by South Carolina regions.

\subsection{Storage and Value of SOC by County, Region, and Soil Order for the State of South Carolina (U.S.A.)}

Soil orders with the highest midpoint storage and value for SOC were: Ultisols (\$64.35B), Histosols (\$11.22B), and Inceptisols (\$10.31B) (Tables 5 and 6). The regions with the highest midpoint storage and SOC values were: Pee Dee (\$34.24B), Low Country (\$32.17B), and Midlands (\$29.24B) (Tables 5 and 6). The counties with the highest midpoint SOC storage and values were Colleton (\$5.44B), Horry (\$5.37B), and Berkeley (\$4.12B) (Tables 5 and 6).

\subsection{Storage and Value of SIC by County, Region, and Soil Order for the State of South} Carolina (U.S.A.)

Soil orders with the highest midpoint storage and value for SIC were: Inceptisols (\$5.91B), Entisols (\$5.53B), and Alfisols (\$5.0B) (Tables 7 and 8). The regions with the highest midpoint SIC storage and values were: Low Country (\$5.69B), Midlands (\$5.55B), and Pee Dee (\$4.67B) (Tables 7 and 8). The counties with the highest midpoint SIC storage and values were Charleston (\$1.46B), Georgetown (\$852.81M), and Horry (\$843.18M) (Tables 7 and 8).

\subsection{Storage and Value of TSC (SOC + SIC) by County, Region, and Soil Order for the State of} South Carolina (U.S.A.)

Soil orders with the highest midpoint storage and value for TSC were: Ultisols (\$64.35B), Inceptisols (\$16.22B), and Entisols (\$14.65B) (Tables 9 and 10). The regions with the highest midpoint TSC storage and values were: Low Country (\$37.86B), Pee Dee (\$36.91B), and Midlands (\$34.79B) (Tables 9 and 10). The counties with the highest midpoint TSC storage and values were Horry (\$6.22B), Colleton (\$6.02B), and Georgetown (\$4.87B) (Tables 9 and 10).

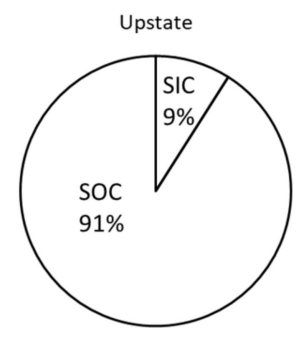

(a)

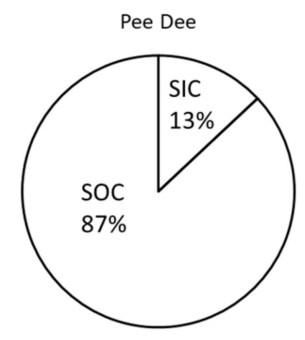

(c)

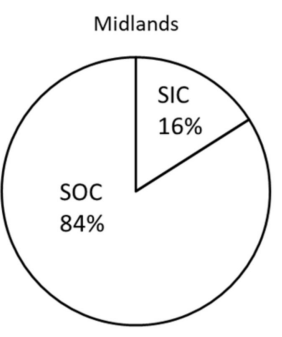

(b)

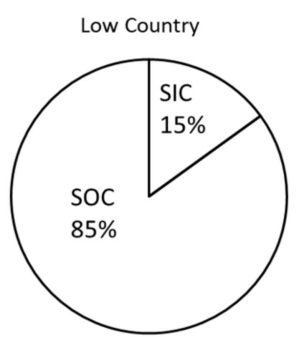

(d)

Figure 3. Distribution of soil carbon by region in the state of South Carolina: (a) Upstate, (b) Midlands, (c) Pee Dee, and (d) Low Country. 
Table 5. Mid-point total soil organic carbon (SOC) storage values by county, region, and soil order for the state of South Carolina (U.S.A.), based on mid-point soil organic carbon (SOC) content numbers in the upper $2 \mathrm{~m}$ of the soil based on data from Guo et al., 2006 [18].

\begin{tabular}{|c|c|c|c|c|c|c|c|c|}
\hline \multirow{4}{*}{$\begin{array}{c}\text { County } \\
\text { (Region) }\end{array}$} & \multirow{4}{*}{$\begin{array}{c}\text { Total Storage } \\
\text { (kg) } \\
\text { (Rank) }\end{array}$} & \multicolumn{7}{|c|}{ Slight $<\longrightarrow$ Degree of Weathering and Soil Development $\longrightarrow$ Strong } \\
\hline & & \multicolumn{3}{|c|}{ Slightly Weathered } & \multicolumn{2}{|c|}{ Moderately Weathered } & \multicolumn{2}{|c|}{ Strongly Weathered } \\
\hline & & Entisols & Inceptisols & Histosols & Alfisols & Mollisols & Spodosols & Ultisols \\
\hline & & \multicolumn{7}{|c|}{ Total Storage (kg) } \\
\hline Anderson & $1.32 \times 10^{10}(19)$ & $8.16 \times 10^{8}$ & 0 & 0 & 0 & 0 & 0 & $1.23 \times 10^{10}$ \\
\hline Cherokee & $7.50 \times 10^{9}(43)$ & $9.44 \times 10^{8}$ & $8.37 \times 10^{8}$ & 0 & $7.50 \times 10^{8}$ & 0 & 0 & $4.97 \times 10^{9}$ \\
\hline Greenville & $1.40 \times 10^{10}(15)$ & $8.64 \times 10^{8}$ & $1.48 \times 10^{9}$ & 0 & 0 & 0 & 0 & $1.17 \times 10^{10}$ \\
\hline Oconee & $1.16 \times 10^{10}(28)$ & $2.96 \times 10^{8}$ & $4.98 \times 10^{8}$ & 0 & 0 & 0 & 0 & $1.08 \times 10^{10}$ \\
\hline Pickens & $9.06 \times 10^{9}(37)$ & $3.12 \times 10^{8}$ & $4.90 \times 10^{8}$ & 0 & 0 & 0 & 0 & $8.26 \times 10^{9}$ \\
\hline Spartanburg & $1.37 \times 10^{10}(16)$ & $6.40 \times 10^{7}$ & $1.43 \times 10^{9}$ & 0 & $5.78 \times 10^{8}$ & 0 & 0 & $1.16 \times 10^{10}$ \\
\hline Union & $9.71 \times 10^{9}(34)$ & $5.92 \times 10^{8}$ & $5.43 \times 10^{8}$ & 0 & $2.73 \times 10^{9}$ & 0 & 0 & $5.84 \times 10^{9}$ \\
\hline (Upstate) & $7.87 \times 10^{10}(4)$ & $3.89 \times 10^{9}$ & $5.28 \times 10^{9}$ & 0 & $4.06 \times 10^{9}$ & 0 & 0 & $6.55 \times 10^{10}$ \\
\hline Abbeville & $9.29 \times 10^{9}(36)$ & $4.16 \times 10^{8}$ & $3.38 \times 10^{8}$ & 0 & $3.01 \times 10^{9}$ & 0 & 0 & $5.52 \times 10^{9}$ \\
\hline Aiken & $2.18 \times 10^{10}(5)$ & $3.56 \times 10^{9}$ & $1.90 \times 10^{9}$ & $1.54 \times 10^{9}$ & $3.75 \times 10^{7}$ & 0 & 0 & $1.48 \times 10^{10}$ \\
\hline Chester & $1.11 \times 10^{10}(30)$ & $4.80 \times 10^{8}$ & $7.30 \times 10^{8}$ & 0 & $4.28 \times 10^{9}$ & $8.10 \times 10^{7}$ & 0 & $5.50 \times 10^{9}$ \\
\hline Edgefield & $9.37 \times 10^{9}(35)$ & $8.08 \times 10^{8}$ & $4.98 \times 10^{8}$ & 0 & $4.58 \times 10^{8}$ & 0 & 0 & $7.60 \times 10^{9}$ \\
\hline Fairfield & $1.25 \times 10^{10}(24)$ & $8.00 \times 10^{6}$ & $1.56 \times 10^{9}$ & 0 & $4.39 \times 10^{9}$ & 0 & 0 & $6.55 \times 10^{9}$ \\
\hline Greenwood & $8.66 \times 10^{9}(39)$ & 0 & $1.03 \times 10^{9}$ & 0 & $2.51 \times 10^{9}$ & 0 & 0 & $5.11 \times 10^{9}$ \\
\hline Kershaw & $1.66 \times 10^{10}(11)$ & $3.06 \times 10^{9}$ & $1.43 \times 10^{9}$ & $2.80 \times 10^{9}$ & $3.53 \times 10^{8}$ & 0 & 0 & $8.95 \times 10^{9}$ \\
\hline Lancaster & $1.03 \times 10^{10}(33)$ & $4.24 \times 10^{8}$ & $1.34 \times 10^{9}$ & 0 & $4.65 \times 10^{8}$ & 0 & 0 & $8.12 \times 10^{9}$ \\
\hline Laurens & $1.33 \times 10^{10}(17)$ & $1.00 \times 10^{9}$ & $1.69 \times 10^{8}$ & 0 & $2.63 \times 10^{9}$ & 0 & 0 & $9.53 \times 10^{9}$ \\
\hline Lexington & $1.31 \times 10^{10}(20)$ & $3.63 \times 10^{9}$ & $7.65 \times 10^{8}$ & 0 & $2.70 \times 10^{8}$ & 0 & $1.23 \times 10^{8}$ & $8.31 \times 10^{9}$ \\
\hline Newberry & $1.18 \times 10^{10}(27)$ & $5.60 \times 10^{8}$ & $5.70 \times 10^{8}$ & 0 & $2.09 \times 10^{9}$ & 0 & 0 & $8.58 \times 10^{9}$ \\
\hline Richland & $1.48 \times 10^{10}(14)$ & $1.30 \times 10^{9}$ & $3.20 \times 10^{9}$ & $1.12 \times 10^{9}$ & $1.43 \times 10^{8}$ & 0 & 0 & $9.07 \times 10^{9}$ \\
\hline Saluda & $8.50 \times 10^{9}(40)$ & $1.52 \times 10^{8}$ & $7.39 \times 10^{8}$ & 0 & $5.78 \times 10^{8}$ & 0 & 0 & $7.04 \times 10^{9}$ \\
\hline York & $1.29 \times 10^{10}(21)$ & $4.00 \times 10^{7}$ & $1.19 \times 10^{9}$ & 0 & $4.33 \times 10^{9}$ & 0 & 0 & $7.36 \times 10^{9}$ \\
\hline (Midlands) & $1.73 \times 10^{11}(3)$ & $1.54 \times 10^{10}$ & $1.55 \times 10^{10}$ & $4.34 \times 10^{9}$ & $2.55 \times 10^{10}$ & $8.10 \times 10^{7}$ & $1.23 \times 10^{8}$ & $1.12 \times 10^{11}$ \\
\hline Chesterfield & $1.59 \times 10^{10}(12)$ & $1.38 \times 10^{9}$ & $5.83 \times 10^{9}$ & 0 & $1.73 \times 10^{8}$ & 0 & 0 & $8.53 \times 10^{9}$ \\
\hline Clarendon & $1.23 \times 10^{10}(25)$ & $3.12 \times 10^{8}$ & $1.71 \times 10^{9}$ & $8.41 \times 10^{8}$ & 0 & 0 & 0 & $9.44 \times 10^{9}$ \\
\hline Darlington & $1.19 \times 10^{10}(26)$ & $2.88 \times 10^{8}$ & $2.30 \times 10^{9}$ & $1.26 \times 10^{9}$ & 0 & 0 & $1.23 \times 10^{7}$ & $8.08 \times 10^{9}$ \\
\hline Dillon & $8.86 \times 10^{9}(38)$ & $7.28 \times 10^{8}$ & $1.14 \times 10^{9}$ & $1.12 \times 10^{9}$ & 0 & 0 & $2.34 \times 10^{8}$ & $5.64 \times 10^{9}$ \\
\hline Florence & $1.50 \times 10^{10}(13)$ & $7.76 \times 10^{8}$ & $1.99 \times 10^{9}$ & 0 & 0 & 0 & $2.46 \times 10^{7}$ & $1.22 \times 10^{10}$ \\
\hline Georgetown & $2.38 \times 10^{10}(4)$ & $2.81 \times 10^{9}$ & $2.44 \times 10^{9}$ & $7.99 \times 10^{9}$ & $3.07 \times 10^{9}$ & 0 & $1.41 \times 10^{9}$ & $6.09 \times 10^{9}$ \\
\hline Horry & $3.19 \times 10^{10}(2)$ & $2.02 \times 10^{9}$ & $3.84 \times 10^{9}$ & $8.97 \times 10^{9}$ & $2.15 \times 10^{9}$ & 0 & $4.06 \times 10^{9}$ & $1.08 \times 10^{10}$ \\
\hline Lee & $7.77 \times 10^{9}(42)$ & $2.32 \times 10^{8}$ & $1.17 \times 10^{9}$ & 0 & $7.50 \times 10^{6}$ & 0 & 0 & $6.37 \times 10^{9}$ \\
\hline Marion & $1.33 \times 10^{10}(18)$ & $8.56 \times 10^{8}$ & $2.55 \times 10^{9}$ & $3.78 \times 10^{9}$ & 0 & 0 & $6.03 \times 10^{8}$ & $5.48 \times 10^{9}$ \\
\hline Marlboro & $2.01 \times 10^{10}(8)$ & $6.00 \times 10^{8}$ & $2.39 \times 10^{9}$ & $1.13 \times 10^{10}$ & $1.28 \times 10^{8}$ & 0 & $2.46 \times 10^{7}$ & $5.58 \times 10^{9}$ \\
\hline Sumter & $1.27 \times 10^{10}(23)$ & $7.20 \times 10^{7}$ & $3.12 \times 10^{9}$ & 0 & $3.00 \times 10^{7}$ & 0 & $2.46 \times 10^{7}$ & $9.44 \times 10^{9}$ \\
\hline Williamsburg & $1.75 \times 10^{10}(10)$ & $2.00 \times 10^{8}$ & $1.86 \times 10^{9}$ & 0 & 0 & 0 & $3.69 \times 10^{7}$ & $1.54 \times 10^{10}$ \\
\hline (Pee Dee) & $1.91 \times 10^{11}(1)$ & $1.03 \times 10^{10}$ & $3.03 \times 10^{10}$ & $3.53 \times 10^{10}$ & $5.56 \times 10^{9}$ & 0 & $6.43 \times 10^{9}$ & $1.03 \times 10^{11}$ \\
\hline Allendale & $8.49 \times 10^{9}(41)$ & $2.00 \times 10^{8}$ & $8.99 \times 10^{8}$ & $8.41 \times 10^{8}$ & 0 & 0 & 0 & $6.55 \times 10^{9}$ \\
\hline Bamberg & $7.38 \times 10^{9}(44)$ & $1.01 \times 10^{9}$ & $8.90 \times 10^{6}$ & 0 & $3.08 \times 10^{8}$ & 0 & $3.69 \times 10^{7}$ & $6.01 \times 10^{9}$ \\
\hline Barnwell & $1.04 \times 10^{10}(32)$ & $6.24 \times 10^{8}$ & $1.23 \times 10^{9}$ & 0 & 0 & 0 & 0 & $8.52 \times 10^{9}$ \\
\hline Beaufort & $1.27 \times 10^{10}(22)$ & $5.58 \times 10^{9}$ & $3.03 \times 10^{8}$ & $8.41 \times 10^{8}$ & $3.00 \times 10^{8}$ & $3.11 \times 10^{8}$ & $2.58 \times 10^{9}$ & $2.78 \times 10^{9}$ \\
\hline Berkeley & $2.44 \times 10^{10}(3)$ & $1.16 \times 10^{9}$ & $1.85 \times 10^{9}$ & $3.22 \times 10^{9}$ & $3.07 \times 10^{9}$ & 0 & $1.69 \times 10^{9}$ & $1.34 \times 10^{10}$ \\
\hline Calhoun & $5.42 \times 10^{9}(46)$ & $5.28 \times 10^{8}$ & $2.31 \times 10^{8}$ & 0 & 0 & 0 & 0 & $4.66 \times 10^{9}$ \\
\hline Charleston & $1.94 \times 10^{10}(9)$ & $6.12 \times 10^{9}$ & $2.95 \times 10^{9}$ & 0 & $5.45 \times 10^{9}$ & 0 & $3.36 \times 10^{9}$ & $1.56 \times 10^{9}$ \\
\hline Colleton & $3.22 \times 10^{10}(1)$ & $2.24 \times 10^{9}$ & $4.36 \times 10^{8}$ & $1.23 \times 10^{10}$ & $1.05 \times 10^{9}$ & $1.15 \times 10^{9}$ & $1.34 \times 10^{9}$ & $1.37 \times 10^{10}$ \\
\hline Dorchester & $1.10 \times 10^{10}(31)$ & $2.19 \times 10^{9}$ & $1.51 \times 10^{8}$ & $1.40 \times 10^{8}$ & $2.10 \times 10^{9}$ & 0 & $3.69 \times 10^{8}$ & $6.06 \times 10^{9}$ \\
\hline Hampton & $1.12 \times 10^{10}(29)$ & $1.09 \times 10^{9}$ & $7.74 \times 10^{8}$ & $4.20 \times 10^{8}$ & $7.58 \times 10^{8}$ & 0 & $4.92 \times 10^{8}$ & $7.64 \times 10^{9}$ \\
\hline Jasper & $2.08 \times 10^{10}(6)$ & $2.54 \times 10^{9}$ & $5.16 \times 10^{8}$ & $7.99 \times 10^{9}$ & $1.85 \times 10^{9}$ & $1.57 \times 10^{9}$ & $2.95 \times 10^{8}$ & $6.04 \times 10^{9}$ \\
\hline McCormick & $6.81 \times 10^{9}(45)$ & $5.12 \times 10^{8}$ & $5.79 \times 10^{8}$ & 0 & $1.85 \times 10^{9}$ & 0 & 0 & $3.87 \times 10^{9}$ \\
\hline Orangeburg & $2.03 \times 10^{10}(7)$ & $5.84 \times 10^{8}$ & $1.60 \times 10^{8}$ & 0 & $1.88 \times 10^{8}$ & 0 & $1.23 \times 10^{7}$ & $1.94 \times 10^{10}$ \\
\hline (Low Country) & $1.90 \times 10^{11}(2)$ & $2.44 \times 10^{10}$ & $1.01 \times 10^{10}$ & $2.58 \times 10^{10}$ & $1.69 \times 10^{10}$ & $3.02 \times 10^{9}$ & $1.02 \times 10^{10}$ & $1.00 \times 10^{11}$ \\
\hline Totals (kg) & $6.34 \times 10^{11}$ & $5.40 \times 10^{10}$ & $6.12 \times 10^{10}$ & $6.65 \times 10^{10}$ & $5.21 \times 10^{10}$ & $3.11 \times 10^{9}$ & $1.67 \times 10^{10}$ & $3.81 \times 10^{11}$ \\
\hline
\end{tabular}


Table 6. The total dollar value of soil organic carbon (SOC) by county, region, and soil order for the state of South Carolina (U.S.A.), based on mid-point soil organic carbon (SOC) numbers for the upper $2 \mathrm{~m}$ from Guo et al. 2006 [18] and a social cost of carbon $\left(\mathrm{SC}-\mathrm{CO}_{2}\right)$ of $\$ 46$ per metric ton of $\mathrm{CO}_{2}$ (2007 U.S. dollars with an average discount rate of 3\% [17]).

\begin{tabular}{|c|c|c|c|c|c|c|c|c|}
\hline \multirow{4}{*}{ County (Region) } & \multirow{4}{*}{$\begin{array}{c}\text { Total Value } \\
\text { (\$) } \\
\text { (Rank) }\end{array}$} & \multicolumn{7}{|c|}{ Slight $<\longrightarrow$ Degree of Weathering and Soil Development $\longrightarrow$ Strong } \\
\hline & & \multicolumn{3}{|c|}{ Slightly Weathered } & \multicolumn{2}{|c|}{ Moderately Weathered } & \multicolumn{2}{|c|}{ Strongly Weathered } \\
\hline & & Entisols & Inceptisols & Histosols & Alfisols & Mollisols & Spodosols & Ultisols \\
\hline & & \multicolumn{7}{|c|}{ Value (\$) } \\
\hline Anderson & $2.22 \times 10^{9}(19)$ & $1.38 \times 10^{8}$ & 0 & 0 & 0 & 0 & 0 & $2.09 \times 10^{9}$ \\
\hline Cherokee & $1.27 \times 10^{9}(43)$ & $1.59 \times 10^{8}$ & $1.41 \times 10^{8}$ & 0 & $1.27 \times 10^{8}$ & 0 & 0 & $8.40 \times 10^{8}$ \\
\hline Greenville & $2.37 \times 10^{9}(15)$ & $1.46 \times 10^{8}$ & $2.49 \times 10^{8}$ & 0 & 0 & 0 & 0 & $1.97 \times 10^{9}$ \\
\hline Oconee & $1.97 \times 10^{9}(28)$ & $5.00 \times 10^{7}$ & $8.40 \times 10^{7}$ & 0 & 0 & 0 & 0 & $1.83 \times 10^{9}$ \\
\hline Pickens & $1.53 \times 10^{9}(37)$ & $5.27 \times 10^{7}$ & $8.25 \times 10^{7}$ & 0 & 0 & 0 & 0 & $1.40 \times 10^{9}$ \\
\hline Spartanburg & $2.31 \times 10^{9}(16)$ & $1.08 \times 10^{7}$ & $2.42 \times 10^{8}$ & 0 & $9.78 \times 10^{7}$ & 0 & 0 & $1.96 \times 10^{9}$ \\
\hline Union & $1.64 \times 10^{9}(34)$ & $9.99 \times 10^{7}$ & $9.15 \times 10^{7}$ & 0 & $4.62 \times 10^{8}$ & 0 & 0 & $9.88 \times 10^{8}$ \\
\hline (Upstate) & $1.33 \times 10^{10}(4)$ & $6.56 \times 10^{8}$ & $8.90 \times 10^{8}$ & 0 & $6.87 \times 10^{8}$ & 0 & 0 & $1.11 \times 10^{10}$ \\
\hline Abbeville & $1.57 \times 10^{9}(36)$ & $7.02 \times 10^{7}$ & $5.70 \times 10^{7}$ & 0 & $5.09 \times 10^{8}$ & 0 & 0 & $9.34 \times 10^{8}$ \\
\hline Aiken & $3.69 \times 10^{9}(5)$ & $6.01 \times 10^{8}$ & $3.20 \times 10^{8}$ & $2.60 \times 10^{8}$ & $6.35 \times 10^{6}$ & 0 & 0 & $2.50 \times 10^{9}$ \\
\hline Chester & $1.87 \times 10^{9}(30)$ & $8.10 \times 10^{7}$ & $1.23 \times 10^{8}$ & 0 & $7.24 \times 10^{8}$ & $1.37 \times 10^{7}$ & 0 & $9.30 \times 10^{8}$ \\
\hline Edgefield & $1.58 \times 10^{9}(35)$ & $1.36 \times 10^{8}$ & $8.40 \times 10^{7}$ & 0 & $7.75 \times 10^{7}$ & 0 & 0 & $1.29 \times 10^{9}$ \\
\hline Fairfield & $2.11 \times 10^{9}(24)$ & $1.35 \times 10^{6}$ & $2.63 \times 10^{8}$ & 0 & $7.43 \times 10^{8}$ & 0 & 0 & $1.11 \times 10^{9}$ \\
\hline Greenwood & $1.46 \times 10^{9}(39)$ & 0 & $1.74 \times 10^{8}$ & 0 & $4.25 \times 10^{8}$ & 0 & 0 & $8.64 \times 10^{8}$ \\
\hline Kershaw & $2.80 \times 10^{9}(11)$ & $5.17 \times 10^{8}$ & $2.42 \times 10^{8}$ & $4.72 \times 10^{8}$ & $5.97 \times 10^{7}$ & 0 & 0 & $1.51 \times 10^{9}$ \\
\hline Lancaster & $1.75 \times 10^{9}(33)$ & $7.16 \times 10^{7}$ & $2.27 \times 10^{8}$ & 0 & $7.87 \times 10^{7}$ & 0 & 0 & $1.37 \times 10^{9}$ \\
\hline Laurens & $2.25 \times 10^{9}(17)$ & $1.69 \times 10^{8}$ & $2.85 \times 10^{7}$ & 0 & $4.46 \times 10^{8}$ & 0 & 0 & $1.61 \times 10^{9}$ \\
\hline Lexington & $2.21 \times 10^{9}(20)$ & $6.13 \times 10^{8}$ & $1.29 \times 10^{8}$ & 0 & $4.57 \times 10^{7}$ & 0 & $2.07 \times 10^{7}$ & $1.40 \times 10^{9}$ \\
\hline Newberry & $1.99 \times 10^{9}(27)$ & $9.45 \times 10^{7}$ & $9.60 \times 10^{7}$ & 0 & $3.53 \times 10^{8}$ & 0 & 0 & $1.45 \times 10^{9}$ \\
\hline Richland & $2.51 \times 10^{9}(14)$ & $2.20 \times 10^{8}$ & $5.40 \times 10^{8}$ & $1.89 \times 10^{8}$ & $2.41 \times 10^{7}$ & 0 & 0 & $1.53 \times 10^{9}$ \\
\hline Saluda & $1.44 \times 10^{9}(40)$ & $2.57 \times 10^{7}$ & $1.25 \times 10^{8}$ & 0 & $9.78 \times 10^{7}$ & 0 & 0 & $1.19 \times 10^{9}$ \\
\hline York & $2.18 \times 10^{9}(21)$ & $6.75 \times 10^{6}$ & $2.01 \times 10^{8}$ & 0 & $7.33 \times 10^{8}$ & 0 & 0 & $1.24 \times 10^{9}$ \\
\hline (Midlands) & $2.92 \times 10^{10}(3)$ & $2.61 \times 10^{9}$ & $2.61 \times 10^{9}$ & $7.32 \times 10^{8}$ & $4.32 \times 10^{9}$ & $1.37 \times 10^{7}$ & $2.07 \times 10^{7}$ & $1.89 \times 10^{10}$ \\
\hline Chesterfield & $2.69 \times 10^{9}(12)$ & $2.34 \times 10^{8}$ & $9.83 \times 10^{8}$ & 0 & $2.92 \times 10^{7}$ & 0 & 0 & $1.44 \times 10^{9}$ \\
\hline Clarendon & $2.08 \times 10^{9}(25)$ & $5.27 \times 10^{7}$ & $2.88 \times 10^{8}$ & $1.42 \times 10^{8}$ & 0 & 0 & 0 & $1.59 \times 10^{9}$ \\
\hline Darlington & $2.02 \times 10^{9}(26)$ & $4.86 \times 10^{7}$ & $3.87 \times 10^{8}$ & $2.13 \times 10^{8}$ & 0 & 0 & $2.07 \times 10^{6}$ & $1.37 \times 10^{9}$ \\
\hline Dillon & $1.50 \times 10^{9}(38)$ & $1.23 \times 10^{8}$ & $1.92 \times 10^{8}$ & $1.89 \times 10^{8}$ & 0 & 0 & $3.93 \times 10^{7}$ & $9.53 \times 10^{8}$ \\
\hline Florence & $2.54 \times 10^{9}(13)$ & $1.31 \times 10^{8}$ & $3.36 \times 10^{8}$ & 0 & 0 & 0 & $4.14 \times 10^{6}$ & $2.07 \times 10^{9}$ \\
\hline Georgetown & $4.02 \times 10^{9}(4)$ & $4.74 \times 10^{8}$ & $4.11 \times 10^{8}$ & $1.35 \times 10^{9}$ & $5.19 \times 10^{8}$ & 0 & $2.38 \times 10^{8}$ & $1.03 \times 10^{9}$ \\
\hline Horry & $5.37 \times 10^{9}(2)$ & $3.40 \times 10^{8}$ & $6.47 \times 10^{8}$ & $1.51 \times 10^{9}$ & $3.64 \times 10^{8}$ & 0 & $6.83 \times 10^{8}$ & $1.83 \times 10^{9}$ \\
\hline Lee & $1.31 \times 10^{9}(42)$ & $3.92 \times 10^{7}$ & $1.97 \times 10^{8}$ & 0 & $1.27 \times 10^{6}$ & 0 & 0 & $1.08 \times 10^{9}$ \\
\hline Marion & $2.24 \times 10^{9}(18)$ & $1.44 \times 10^{8}$ & $4.29 \times 10^{8}$ & $6.38 \times 10^{8}$ & 0 & 0 & $1.01 \times 10^{8}$ & $9.26 \times 10^{8}$ \\
\hline Marlboro & $3.39 \times 10^{9}(8)$ & $1.01 \times 10^{8}$ & $4.04 \times 10^{8}$ & $1.91 \times 10^{9}$ & $2.16 \times 10^{7}$ & 0 & $4.14 \times 10^{6}$ & $9.43 \times 10^{8}$ \\
\hline Sumter & $2.14 \times 10^{9}(23)$ & $1.22 \times 10^{7}$ & $5.25 \times 10^{8}$ & 0 & $5.08 \times 10^{6}$ & 0 & $4.14 \times 10^{6}$ & $1.59 \times 10^{9}$ \\
\hline Williamsburg & $2.95 \times 10^{9}(10)$ & $3.38 \times 10^{7}$ & $3.14 \times 10^{8}$ & 0 & 0 & 0 & $6.21 \times 10^{6}$ & $2.60 \times 10^{9}$ \\
\hline (Pee Dee) & $3.22 \times 10^{10}(1)$ & $1.73 \times 10^{9}$ & $5.11 \times 10^{9}$ & $5.95 \times 10^{9}$ & $9.41 \times 10^{8}$ & 0 & $1.08 \times 10^{9}$ & $1.74 \times 10^{10}$ \\
\hline Allendale & $1.43 \times 10^{9}(41)$ & $3.38 \times 10^{7}$ & $1.52 \times 10^{8}$ & $1.42 \times 10^{8}$ & 0 & 0 & 0 & $1.11 \times 10^{9}$ \\
\hline Bamberg & $1.25 \times 10^{9}(44)$ & $1.70 \times 10^{8}$ & $1.50 \times 10^{6}$ & 0 & $5.21 \times 10^{7}$ & 0 & $6.21 \times 10^{6}$ & $1.02 \times 10^{9}$ \\
\hline Barnwell & $1.75 \times 10^{9}(32)$ & $1.05 \times 10^{8}$ & $2.07 \times 10^{8}$ & 0 & 0 & 0 & 0 & $1.44 \times 10^{9}$ \\
\hline Beaufort & $2.14 \times 10^{9}(22)$ & $9.42 \times 10^{8}$ & $5.10 \times 10^{7}$ & $1.42 \times 10^{8}$ & $5.08 \times 10^{7}$ & $5.24 \times 10^{7}$ & $4.35 \times 10^{8}$ & $4.69 \times 10^{8}$ \\
\hline Berkeley & $4.12 \times 10^{9}(3)$ & $1.96 \times 10^{8}$ & $3.12 \times 10^{8}$ & $5.43 \times 10^{8}$ & $5.19 \times 10^{8}$ & 0 & $2.84 \times 10^{8}$ & $2.26 \times 10^{9}$ \\
\hline Calhoun & $9.15 \times 10^{8}(46)$ & $8.91 \times 10^{7}$ & $3.90 \times 10^{7}$ & 0 & 0 & 0 & 0 & $7.87 \times 10^{8}$ \\
\hline Charleston & $3.28 \times 10^{9}(9)$ & $1.03 \times 10^{9}$ & $4.98 \times 10^{8}$ & 0 & $9.23 \times 10^{8}$ & 0 & $5.65 \times 10^{8}$ & $2.64 \times 10^{8}$ \\
\hline Colleton & $5.44 \times 10^{9}(1)$ & $3.78 \times 10^{8}$ & $7.35 \times 10^{7}$ & $2.08 \times 10^{9}$ & $1.78 \times 10^{8}$ & $1.94 \times 10^{8}$ & $2.26 \times 10^{8}$ & $2.31 \times 10^{9}$ \\
\hline Dorchester & $1.86 \times 10^{9}(31)$ & $3.70 \times 10^{8}$ & $2.55 \times 10^{7}$ & $2.36 \times 10^{7}$ & $3.56 \times 10^{8}$ & 0 & $6.21 \times 10^{7}$ & $1.02 \times 10^{9}$ \\
\hline Hampton & $1.89 \times 10^{9}(29)$ & $1.84 \times 10^{8}$ & $1.31 \times 10^{8}$ & $7.09 \times 10^{7}$ & $1.28 \times 10^{8}$ & 0 & $8.28 \times 10^{7}$ & $1.29 \times 10^{9}$ \\
\hline Jasper & $3.51 \times 10^{9}(6)$ & $4.29 \times 10^{8}$ & $8.70 \times 10^{7}$ & $1.35 \times 10^{9}$ & $3.12 \times 10^{8}$ & $2.64 \times 10^{8}$ & $4.97 \times 10^{7}$ & $1.02 \times 10^{9}$ \\
\hline McCormick & $1.15 \times 10^{9}(45)$ & $8.64 \times 10^{7}$ & $9.75 \times 10^{7}$ & 0 & $3.14 \times 10^{8}$ & 0 & 0 & $6.54 \times 10^{8}$ \\
\hline Orangeburg & $3.43 \times 10^{9}(7)$ & $9.86 \times 10^{7}$ & $2.70 \times 10^{7}$ & 0 & $3.18 \times 10^{7}$ & 0 & $2.07 \times 10^{6}$ & $3.27 \times 10^{9}$ \\
\hline (Low Country) & $3.22 \times 10^{10}(2)$ & $4.11 \times 10^{9}$ & $1.70 \times 10^{9}$ & $4.35 \times 10^{9}$ & $2.87 \times 10^{9}$ & $5.11 \times 10^{8}$ & $1.71 \times 10^{9}$ & $1.69 \times 10^{10}$ \\
\hline Totals (\$) & $1.07 \times 10^{11}$ & $9.11 \times 10^{9}$ & $1.03 \times 10^{10}$ & $1.12 \times 10^{10}$ & $8.82 \times 10^{9}$ & $5.24 \times 10^{8}$ & $2.82 \times 10^{9}$ & $6.43 \times 10^{10}$ \\
\hline
\end{tabular}


Table 7. Mid-point total soil inorganic carbon (SIC) storage by county, region, and soil order for the state of South Carolina (U.S.A.), based on mid-point soil inorganic carbon (SIC) contents in the upper $2 \mathrm{~m}$ based on data from Guo et al., 2006 [18].

\begin{tabular}{|c|c|c|c|c|c|c|c|c|}
\hline \multirow{4}{*}{$\begin{array}{l}\text { County } \\
\text { (Region) }\end{array}$} & \multirow{4}{*}{$\begin{array}{c}\text { Total Storage } \\
\text { (kg) } \\
\text { (Rank) }\end{array}$} & \multicolumn{7}{|c|}{ Slight $<\longrightarrow$ Degree of Weathering and Soil Development $\longrightarrow$ Strong } \\
\hline & & \multicolumn{3}{|c|}{ Slightly Weathered } & \multicolumn{2}{|c|}{ Moderately Weathered } & \multicolumn{2}{|c|}{ Strongly Weathered } \\
\hline & & Entisols & Inceptisols & Histosols & Alfisols & Mollisols & Spodosols & Ultisols \\
\hline & & \multicolumn{7}{|c|}{ Total Storage (kg) } \\
\hline Anderson & $4.90 \times 10^{8}(43)$ & $4.90 \times 10^{8}$ & 0 & 0 & 0 & 0 & 0 & 0 \\
\hline Cherokee & $1.48 \times 10^{9}(29)$ & $5.66 \times 10^{8}$ & $4.79 \times 10^{8}$ & 0 & $4.30 \times 10^{8}$ & 0 & 0 & 0 \\
\hline Greenville & $1.37 \times 10^{9}(30)$ & $5.18 \times 10^{8}$ & $8.47 \times 10^{8}$ & 0 & 0 & 0 & 0 & 0 \\
\hline Oconee & $4.63 \times 10^{8}(45)$ & $1.78 \times 10^{8}$ & $2.86 \times 10^{8}$ & 0 & 0 & 0 & 0 & 0 \\
\hline Pickens & $4.68 \times 10^{8}(44)$ & $1.87 \times 10^{8}$ & $2.81 \times 10^{8}$ & 0 & 0 & 0 & 0 & 0 \\
\hline Spartanburg & $1.19 \times 10^{9}(32)$ & $3.84 \times 10^{7}$ & $8.21 \times 10^{8}$ & 0 & $3.31 \times 10^{8}$ & 0 & 0 & 0 \\
\hline Union & $2.23 \times 10^{9}(17)$ & $3.55 \times 10^{8}$ & $3.11 \times 10^{8}$ & 0 & $1.57 \times 10^{9}$ & 0 & 0 & 0 \\
\hline (Upstate) & $7.68 \times 10^{9}(4)$ & $2.33 \times 10^{9}$ & $3.02 \times 10^{9}$ & 0 & $2.33 \times 10^{9}$ & 0 & 0 & 0 \\
\hline Abbeville & $2.17 \times 10^{9}(19)$ & $2.50 \times 10^{8}$ & $1.94 \times 10^{8}$ & 0 & $1.72 \times 10^{9}$ & 0 & 0 & 0 \\
\hline Aiken & $3.27 \times 10^{9}(10)$ & $2.14 \times 10^{9}$ & $1.09 \times 10^{9}$ & $2.64 \times 10^{7}$ & $2.15 \times 10^{7}$ & 0 & 0 & 0 \\
\hline Chester & $3.23 \times 10^{9}(11)$ & $2.88 \times 10^{8}$ & $4.18 \times 10^{8}$ & 0 & $2.45 \times 10^{9}$ & $6.90 \times 10^{7}$ & 0 & 0 \\
\hline Edgefield & $1.03 \times 10^{9}(37)$ & $4.85 \times 10^{8}$ & $2.86 \times 10^{8}$ & 0 & $2.62 \times 10^{8}$ & 0 & 0 & 0 \\
\hline Fairfield & $3.41 \times 10^{9}(9)$ & $4.80 \times 10^{6}$ & $8.93 \times 10^{8}$ & 0 & $2.52 \times 10^{9}$ & 0 & 0 & 0 \\
\hline Greenwood & $2.03 \times 10^{9}(21)$ & 0 & $5.92 \times 10^{8}$ & 0 & $1.44 \times 10^{9}$ & 0 & 0 & 0 \\
\hline Kershaw & $2.91 \times 10^{9}(13)$ & $1.84 \times 10^{9}$ & $8.21 \times 10^{8}$ & $4.80 \times 10^{7}$ & $2.02 \times 10^{8}$ & 0 & 0 & 0 \\
\hline Lancaster & $1.29 \times 10^{9}(31)$ & $2.54 \times 10^{8}$ & $7.70 \times 10^{8}$ & 0 & $2.67 \times 10^{8}$ & 0 & 0 & 0 \\
\hline Laurens & $2.21 \times 10^{9}(18)$ & $6.00 \times 10^{8}$ & $9.69 \times 10^{7}$ & 0 & $1.51 \times 10^{9}$ & 0 & 0 & 0 \\
\hline Lexington & $2.78 \times 10^{9}(14)$ & $2.18 \times 10^{9}$ & $4.39 \times 10^{8}$ & 0 & $1.55 \times 10^{8}$ & 0 & $6.00 \times 10^{6}$ & 0 \\
\hline Newberry & $1.86 \times 10^{9}(23)$ & $3.36 \times 10^{8}$ & $3.26 \times 10^{8}$ & 0 & $1.20 \times 10^{9}$ & 0 & 0 & 0 \\
\hline Richland & $2.72 \times 10^{9}(15)$ & $7.82 \times 10^{8}$ & $1.84 \times 10^{9}$ & $1.92 \times 10^{7}$ & $8.17 \times 10^{7}$ & 0 & 0 & 0 \\
\hline Saluda & $8.46 \times 10^{8}(38)$ & $9.12 \times 10^{7}$ & $4.23 \times 10^{8}$ & 0 & $3.31 \times 10^{8}$ & 0 & 0 & 0 \\
\hline York & $3.19 \times 10^{9}(12)$ & $2.40 \times 10^{7}$ & $6.83 \times 10^{8}$ & 0 & $2.48 \times 10^{9}$ & 0 & 0 & 0 \\
\hline (Midlands) & $3.29 \times 10^{10}(2)$ & $9.27 \times 10^{9}$ & $8.86 \times 10^{9}$ & $7.44 \times 10^{7}$ & $1.46 \times 10^{10}$ & $6.90 \times 10^{7}$ & $6.00 \times 10^{6}$ & 0 \\
\hline Chesterfield & $4.27 \times 10^{9}(5)$ & $8.30 \times 10^{8}$ & $3.34 \times 10^{9}$ & 0 & $9.89 \times 10^{7}$ & 0 & 0 & 0 \\
\hline Clarendon & $1.18 \times 10^{9}(34)$ & $1.87 \times 10^{8}$ & $9.79 \times 10^{8}$ & $1.44 \times 10^{7}$ & 0 & 0 & 0 & 0 \\
\hline Darlington & $1.51 \times 10^{9}(28)$ & $1.73 \times 10^{8}$ & $1.32 \times 10^{9}$ & $2.16 \times 10^{7}$ & 0 & 0 & $6.00 \times 10^{5}$ & 0 \\
\hline Dillon & $1.12 \times 10^{9}(35)$ & $4.37 \times 10^{8}$ & $6.53 \times 10^{8}$ & $1.92 \times 10^{7}$ & 0 & 0 & $1.14 \times 10^{7}$ & 0 \\
\hline Florence & $1.61 \times 10^{9}(26)$ & $4.66 \times 10^{8}$ & $1.14 \times 10^{9}$ & 0 & 0 & 0 & $1.20 \times 10^{6}$ & 0 \\
\hline Georgetown & $5.05 \times 10^{9}(2)$ & $1.68 \times 10^{9}$ & $1.40 \times 10^{9}$ & $1.37 \times 10^{8}$ & $1.76 \times 10^{9}$ & 0 & $6.90 \times 10^{7}$ & 0 \\
\hline Horry & $4.99 \times 10^{9}(3)$ & $1.21 \times 10^{9}$ & $2.20 \times 10^{9}$ & $1.54 \times 10^{8}$ & $1.23 \times 10^{9}$ & 0 & $1.98 \times 10^{8}$ & 0 \\
\hline Lee & $8.12 \times 10^{8}(39)$ & $1.39 \times 10^{8}$ & $6.68 \times 10^{8}$ & 0 & $4.30 \times 10^{6}$ & 0 & 0 & 0 \\
\hline Marion & $2.07 \times 10^{9}(20)$ & $5.14 \times 10^{8}$ & $1.46 \times 10^{9}$ & $6.48 \times 10^{7}$ & 0 & 0 & $2.94 \times 10^{7}$ & 0 \\
\hline Marlboro & $2.00 \times 10^{9}(22)$ & $3.60 \times 10^{8}$ & $1.37 \times 10^{9}$ & $1.94 \times 10^{8}$ & $7.31 \times 10^{7}$ & 0 & $1.20 \times 10^{6}$ & 0 \\
\hline Sumter & $1.85 \times 10^{9}(24)$ & $4.32 \times 10^{7}$ & $1.79 \times 10^{9}$ & 0 & $1.72 \times 10^{7}$ & 0 & $1.20 \times 10^{6}$ & 0 \\
\hline Williamsburg & $1.19 \times 10^{9}(33)$ & $1.20 \times 10^{8}$ & $1.07 \times 10^{9}$ & 0 & 0 & 0 & $1.80 \times 10^{6}$ & 0 \\
\hline (Pee Dee) & $2.76 \times 10^{10}(3)$ & $6.16 \times 10^{9}$ & $1.74 \times 10^{10}$ & $6.05 \times 10^{8}$ & $3.19 \times 10^{9}$ & 0 & $3.14 \times 10^{8}$ & 0 \\
\hline Allendale & $6.50 \times 10^{8}(41)$ & $1.20 \times 10^{8}$ & $5.15 \times 10^{8}$ & $1.44 \times 10^{7}$ & 0 & 0 & 0 & 0 \\
\hline Bamberg & $7.88 \times 10^{8}(40)$ & $6.05 \times 10^{8}$ & $5.10 \times 10^{6}$ & 0 & $1.76 \times 10^{8}$ & 0 & $1.80 \times 10^{6}$ & 0 \\
\hline Barnwell & $1.08 \times 10^{9}(36)$ & $3.74 \times 10^{8}$ & $7.04 \times 10^{8}$ & 0 & 0 & 0 & 0 & 0 \\
\hline Beaufort & $4.10 \times 10^{9}(6)$ & $3.35 \times 10^{9}$ & $1.73 \times 10^{8}$ & $1.44 \times 10^{7}$ & $1.72 \times 10^{8}$ & $2.65 \times 10^{8}$ & $1.26 \times 10^{8}$ & 0 \\
\hline Berkeley & $3.65 \times 10^{9}(7)$ & $6.96 \times 10^{8}$ & $1.06 \times 10^{9}$ & $5.52 \times 10^{7}$ & $1.76 \times 10^{9}$ & 0 & $8.22 \times 10^{7}$ & 0 \\
\hline Calhoun & $4.49 \times 10^{8}(46)$ & $3.17 \times 10^{8}$ & $1.33 \times 10^{8}$ & 0 & 0 & 0 & 0 & 0 \\
\hline Charleston & $8.66 \times 10^{9}(1)$ & $3.67 \times 10^{9}$ & $1.69 \times 10^{9}$ & 0 & $3.13 \times 10^{9}$ & 0 & $1.64 \times 10^{8}$ & 0 \\
\hline Colleton & $3.45 \times 10^{9}(8)$ & $1.34 \times 10^{9}$ & $2.50 \times 10^{8}$ & $2.11 \times 10^{8}$ & $6.02 \times 10^{8}$ & $9.78 \times 10^{8}$ & $6.54 \times 10^{7}$ & 0 \\
\hline Dorchester & $2.63 \times 10^{9}(16)$ & $1.32 \times 10^{9}$ & $8.67 \times 10^{7}$ & $2.40 \times 10^{6}$ & $1.20 \times 10^{9}$ & 0 & $1.80 \times 10^{7}$ & 0 \\
\hline Hampton & $1.56 \times 10^{9}(27)$ & $6.53 \times 10^{8}$ & $4.44 \times 10^{8}$ & $7.20 \times 10^{6}$ & $4.34 \times 10^{8}$ & 0 & $2.40 \times 10^{7}$ & 0 \\
\hline Jasper & $4.37 \times 10^{9}(4)$ & $1.53 \times 10^{9}$ & $2.96 \times 10^{8}$ & $1.37 \times 10^{8}$ & $1.06 \times 10^{9}$ & $1.33 \times 10^{9}$ & $1.44 \times 10^{7}$ & 0 \\
\hline McCormick & $1.70 \times 10^{9}(25)$ & $3.07 \times 10^{8}$ & $3.32 \times 10^{8}$ & 0 & $1.06 \times 10^{9}$ & 0 & 0 & 0 \\
\hline Orangeburg & $5.50 \times 10^{8}(42)$ & $3.50 \times 10^{8}$ & $9.18 \times 10^{7}$ & 0 & $1.08 \times 10^{8}$ & 0 & $6.00 \times 10^{5}$ & 0 \\
\hline (Low Country) & $3.36 \times 10^{10}(1)$ & $1.46 \times 10^{10}$ & $5.78 \times 10^{9}$ & $4.42 \times 10^{8}$ & $9.70 \times 10^{9}$ & $2.58 \times 10^{9}$ & $4.96 \times 10^{8}$ & 0 \\
\hline Totals (kg) & $1.02 \times 10^{11}$ & $3.24 \times 10^{10}$ & $3.50 \times 10^{10}$ & $1.14 \times 10^{9}$ & $2.99 \times 10^{10}$ & $2.65 \times 10^{9}$ & $8.16 \times 10^{8}$ & 0 \\
\hline
\end{tabular}


Table 8. The total dollar value of soil inorganic carbon (SIC) by county, region, and soil order for the state of South Carolina (U.S.A.), based on mid-point soil inorganic carbon (SIC) numbers for the upper $2 \mathrm{~m}$ from Guo et al. 2006 [18] and a social cost of carbon $\left(\mathrm{SC}-\mathrm{CO}_{2}\right.$ ) of $\$ 46$ per metric ton of $\mathrm{CO}_{2}$ (2007 U.S. dollars with an average discount rate of 3\% [17]).

\begin{tabular}{|c|c|c|c|c|c|c|c|c|}
\hline \multirow{4}{*}{ County (Region) } & \multirow{4}{*}{$\begin{array}{c}\text { Total Value } \\
\text { (\$) } \\
\text { (Rank) }\end{array}$} & \multicolumn{7}{|c|}{ Slight $<\longrightarrow \longrightarrow$ Degree of Weathering and Soil Development $\longrightarrow$ Strong } \\
\hline & & \multicolumn{3}{|c|}{ Slightly Weathered } & \multicolumn{2}{|c|}{ Moderately Weathered } & \multicolumn{2}{|c|}{ Strongly Weathered } \\
\hline & & Entisols & Inceptisols & Histosols & Alfisols & Mollisols & Spodosols & Ultisols \\
\hline & & \multicolumn{7}{|c|}{ Value (\$) } \\
\hline Anderson & $8.36 \times 10^{7}(43)$ & $8.36 \times 10^{7}$ & 0 & 0 & 0 & 0 & 0 & 0 \\
\hline Cherokee & $2.50 \times 10^{8}(29)$ & $9.68 \times 10^{7}$ & $8.08 \times 10^{7}$ & 0 & $7.20 \times 10^{7}$ & 0 & 0 & 0 \\
\hline Greenville & $2.31 \times 10^{8}(30)$ & $8.86 \times 10^{7}$ & $1.43 \times 10^{8}$ & 0 & 0 & 0 & 0 & 0 \\
\hline Oconee & $7.85 \times 10^{7}(45)$ & $3.03 \times 10^{7}$ & $4.82 \times 10^{7}$ & 0 & 0 & 0 & 0 & 0 \\
\hline Pickens & $7.93 \times 10^{7}(44)$ & $3.20 \times 10^{7}$ & $4.73 \times 10^{7}$ & 0 & 0 & 0 & 0 & 0 \\
\hline Spartanburg & $2.00 \times 10^{8}(32)$ & $6.56 \times 10^{6}$ & $1.38 \times 10^{8}$ & 0 & $5.54 \times 10^{7}$ & 0 & 0 & 0 \\
\hline Union & $3.75 \times 10^{8}(17)$ & $6.07 \times 10^{7}$ & $5.25 \times 10^{7}$ & 0 & $2.62 \times 10^{8}$ & 0 & 0 & 0 \\
\hline (Upstate) & $1.30 \times 10^{9}(4)$ & $3.99 \times 10^{8}$ & $5.10 \times 10^{8}$ & 0 & $3.90 \times 10^{8}$ & 0 & 0 & 0 \\
\hline Abbeville & $3.64 \times 10^{8}(19)$ & $4.26 \times 10^{7}$ & $3.27 \times 10^{7}$ & 0 & $2.89 \times 10^{8}$ & 0 & 0 & 0 \\
\hline Aiken & $5.56 \times 10^{8}(10)$ & $3.65 \times 10^{8}$ & $1.83 \times 10^{8}$ & $4.51 \times 10^{6}$ & $3.60 \times 10^{6}$ & 0 & 0 & 0 \\
\hline Chester & $5.42 \times 10^{8}(11)$ & $4.92 \times 10^{7}$ & $7.05 \times 10^{7}$ & 0 & $4.10 \times 10^{8}$ & $1.16 \times 10^{7}$ & 0 & 0 \\
\hline Edgefield & $1.75 \times 10^{8}(37)$ & $8.28 \times 10^{7}$ & $4.82 \times 10^{7}$ & 0 & $4.39 \times 10^{7}$ & 0 & 0 & 0 \\
\hline Fairfield & $5.73 \times 10^{8}(9)$ & $8.20 \times 10^{5}$ & $1.51 \times 10^{8}$ & 0 & $4.21 \times 10^{8}$ & 0 & 0 & 0 \\
\hline Greenwood & $3.41 \times 10^{8}(21)$ & 0 & $9.98 \times 10^{7}$ & 0 & $2.41 \times 10^{8}$ & 0 & 0 & 0 \\
\hline Kershaw & $4.95 \times 10^{8}(13)$ & $3.14 \times 10^{8}$ & $1.38 \times 10^{8}$ & $8.20 \times 10^{6}$ & $3.38 \times 10^{7}$ & 0 & 0 & 0 \\
\hline Lancaster & $2.18 \times 10^{8}(31)$ & $4.35 \times 10^{7}$ & $1.30 \times 10^{8}$ & 0 & $4.46 \times 10^{7}$ & 0 & 0 & 0 \\
\hline Laurens & $3.72 \times 10^{8}(18)$ & $1.03 \times 10^{8}$ & $1.63 \times 10^{7}$ & 0 & $2.53 \times 10^{8}$ & 0 & 0 & 0 \\
\hline Lexington & $4.73 \times 10^{8}(14)$ & $3.72 \times 10^{8}$ & $7.40 \times 10^{7}$ & 0 & $2.59 \times 10^{7}$ & 0 & $1.00 \times 10^{6}$ & 0 \\
\hline Newberry & $3.13 \times 10^{8}(23)$ & $5.74 \times 10^{7}$ & $5.50 \times 10^{7}$ & 0 & $2.00 \times 10^{8}$ & 0 & 0 & 0 \\
\hline Richland & $4.60 \times 10^{8}(15)$ & $1.34 \times 10^{8}$ & $3.10 \times 10^{8}$ & $3.28 \times 10^{6}$ & $1.37 \times 10^{7}$ & 0 & 0 & 0 \\
\hline Saluda & $1.42 \times 10^{8}(38)$ & $1.56 \times 10^{7}$ & $7.14 \times 10^{7}$ & 0 & $5.54 \times 10^{7}$ & 0 & 0 & 0 \\
\hline York & $5.35 \times 10^{8}(12)$ & $4.10 \times 10^{6}$ & $1.15 \times 10^{8}$ & 0 & $4.15 \times 10^{8}$ & 0 & 0 & 0 \\
\hline (Midlands) & $5.55 \times 10^{9}(2)$ & $1.58 \times 10^{9}$ & $1.49 \times 10^{9}$ & $1.27 \times 10^{7}$ & $2.45 \times 10^{9}$ & $1.16 \times 10^{7}$ & $1.00 \times 10^{6}$ & 0 \\
\hline Chesterfield & $7.22 \times 10^{8}(5)$ & $1.42 \times 10^{8}$ & $5.63 \times 10^{8}$ & 0 & $1.66 \times 10^{7}$ & 0 & 0 & 0 \\
\hline Clarendon & $2.00 \times 10^{8}(34)$ & $3.20 \times 10^{7}$ & $1.65 \times 10^{8}$ & $2.46 \times 10^{6}$ & 0 & 0 & 0 & 0 \\
\hline Darlington & $2.55 \times 10^{8}(28)$ & $2.95 \times 10^{7}$ & $2.22 \times 10^{8}$ & $3.69 \times 10^{6}$ & 0 & 0 & $1.00 \times 10^{5}$ & 0 \\
\hline Dillon & $1.90 \times 10^{8}(35)$ & $7.46 \times 10^{7}$ & $1.10 \times 10^{8}$ & $3.28 \times 10^{6}$ & 0 & 0 & $1.90 \times 10^{6}$ & 0 \\
\hline Florence & $2.72 \times 10^{8}(26)$ & $7.95 \times 10^{7}$ & $1.93 \times 10^{8}$ & 0 & 0 & 0 & $2.00 \times 10^{5}$ & 0 \\
\hline Georgetown & $8.53 \times 10^{8}(2)$ & $2.88 \times 10^{8}$ & $2.36 \times 10^{8}$ & $2.34 \times 10^{7}$ & $2.94 \times 10^{8}$ & 0 & $1.15 \times 10^{7}$ & 0 \\
\hline Horry & $8.43 \times 10^{8}(3)$ & $2.07 \times 10^{8}$ & $3.71 \times 10^{8}$ & $2.62 \times 10^{7}$ & $2.07 \times 10^{8}$ & 0 & $3.30 \times 10^{7}$ & 0 \\
\hline Lee & $1.37 \times 10^{8}(39)$ & $2.38 \times 10^{7}$ & $1.13 \times 10^{8}$ & 0 & $7.20 \times 10^{5}$ & 0 & 0 & 0 \\
\hline Marion & $3.50 \times 10^{8}(20)$ & $8.77 \times 10^{7}$ & $2.46 \times 10^{8}$ & $1.11 \times 10^{7}$ & 0 & 0 & $4.90 \times 10^{6}$ & 0 \\
\hline Marlboro & $3.38 \times 10^{8}(22)$ & $6.15 \times 10^{7}$ & $2.31 \times 10^{8}$ & $3.32 \times 10^{7}$ & $1.22 \times 10^{7}$ & 0 & $2.00 \times 10^{5}$ & 0 \\
\hline Sumter & $3.11 \times 10^{8}(24)$ & $7.38 \times 10^{6}$ & $3.01 \times 10^{8}$ & 0 & $2.88 \times 10^{6}$ & 0 & $2.00 \times 10^{5}$ & 0 \\
\hline Williamsburg & $2.01 \times 10^{8}(33)$ & $2.05 \times 10^{7}$ & $1.80 \times 10^{8}$ & 0 & 0 & 0 & $3.00 \times 10^{5}$ & 0 \\
\hline (Pee Dee) & $4.67 \times 10^{9}(3)$ & $1.05 \times 10^{9}$ & $2.93 \times 10^{9}$ & $1.03 \times 10^{8}$ & $5.34 \times 10^{8}$ & 0 & $5.23 \times 10^{7}$ & 0 \\
\hline Allendale & $1.10 \times 10^{8}(41)$ & $2.05 \times 10^{7}$ & $8.69 \times 10^{7}$ & $2.46 \times 10^{6}$ & 0 & 0 & 0 & 0 \\
\hline Bamberg & $1.34 \times 10^{8}(40)$ & $1.03 \times 10^{8}$ & $8.60 \times 10^{5}$ & 0 & $2.95 \times 10^{7}$ & 0 & $3.00 \times 10^{5}$ & 0 \\
\hline Barnwell & $1.83 \times 10^{8}(36)$ & $6.40 \times 10^{7}$ & $1.19 \times 10^{8}$ & 0 & 0 & 0 & 0 & 0 \\
\hline Beaufort & $6.98 \times 10^{8}(6)$ & $5.72 \times 10^{8}$ & $2.92 \times 10^{7}$ & $2.46 \times 10^{6}$ & $2.88 \times 10^{7}$ & $4.44 \times 10^{7}$ & $2.10 \times 10^{7}$ & 0 \\
\hline Berkeley & $6.15 \times 10^{8}(7)$ & $1.19 \times 10^{8}$ & $1.79 \times 10^{8}$ & $9.43 \times 10^{6}$ & $2.94 \times 10^{8}$ & 0 & $1.37 \times 10^{7}$ & 0 \\
\hline Calhoun & $7.65 \times 10^{7}(46)$ & $5.41 \times 10^{7}$ & $2.24 \times 10^{7}$ & 0 & 0 & 0 & 0 & 0 \\
\hline Charleston & $1.46 \times 10^{9}(1)$ & $6.27 \times 10^{8}$ & $2.86 \times 10^{8}$ & 0 & $5.23 \times 10^{8}$ & 0 & $2.73 \times 10^{7}$ & 0 \\
\hline Colleton & $5.84 \times 10^{8}(8)$ & $2.30 \times 10^{8}$ & $4.21 \times 10^{7}$ & $3.61 \times 10^{7}$ & $1.01 \times 10^{8}$ & $1.64 \times 10^{8}$ & $1.09 \times 10^{7}$ & 0 \\
\hline Dorchester & $4.44 \times 10^{8}(16)$ & $2.25 \times 10^{8}$ & $1.46 \times 10^{7}$ & $4.10 \times 10^{5}$ & $2.02 \times 10^{8}$ & 0 & $3.00 \times 10^{6}$ & 0 \\
\hline Hampton & $2.64 \times 10^{8}(27)$ & $1.12 \times 10^{8}$ & $7.48 \times 10^{7}$ & $1.23 \times 10^{6}$ & $7.27 \times 10^{7}$ & 0 & $4.00 \times 10^{6}$ & 0 \\
\hline Jasper & $7.37 \times 10^{8}(4)$ & $2.61 \times 10^{8}$ & $4.99 \times 10^{7}$ & $2.34 \times 10^{7}$ & $1.77 \times 10^{8}$ & $2.24 \times 10^{8}$ & $2.40 \times 10^{6}$ & 0 \\
\hline McCormick & $2.86 \times 10^{8}(25)$ & $5.25 \times 10^{7}$ & $5.59 \times 10^{7}$ & 0 & $1.78 \times 10^{8}$ & 0 & 0 & 0 \\
\hline Orangeburg & $9.34 \times 10^{7}(42)$ & $5.99 \times 10^{7}$ & $1.55 \times 10^{7}$ & 0 & $1.80 \times 10^{7}$ & 0 & $1.00 \times 10^{5}$ & 0 \\
\hline (Low Country) & $5.69 \times 10^{9}(1)$ & $2.50 \times 10^{9}$ & $9.75 \times 10^{8}$ & $7.54 \times 10^{7}$ & $1.62 \times 10^{9}$ & $4.32 \times 10^{8}$ & $8.27 \times 10^{7}$ & 0 \\
\hline Totals (\$) & $1.72 \times 10^{10}$ & $5.53 \times 10^{9}$ & $5.91 \times 10^{9}$ & $1.95 \times 10^{8}$ & $5.00 \times 10^{9}$ & $4.44 \times 10^{8}$ & $1.36 \times 10^{8}$ & 0 \\
\hline
\end{tabular}


Table 9. Mid-point total soil carbon (TSC) storage by county, region, and soil order for the state of South Carolina (U.S.A.), based on mid-point (TSC) contents in the upper $2 \mathrm{~m}$ based on data from Guo et al. 2006 [18].

\begin{tabular}{|c|c|c|c|c|c|c|c|c|}
\hline \multirow{4}{*}{$\begin{array}{l}\text { County } \\
\text { (Region) }\end{array}$} & \multirow{4}{*}{$\begin{array}{c}\text { Total Storage } \\
\text { (kg) } \\
\text { (Rank) }\end{array}$} & \multicolumn{7}{|c|}{ Slight $<\longrightarrow$ Degree of Weathering and Soil Development $\longrightarrow$ Strong } \\
\hline & & \multicolumn{3}{|c|}{ Slightly Weathered } & \multicolumn{2}{|c|}{ Moderately Weathered } & \multicolumn{2}{|c|}{ Strongly Weathered } \\
\hline & & Entisols & Inceptisols & Histosols & Alfisols & Mollisols & Spodosols & Ultisols \\
\hline & & \multicolumn{7}{|c|}{ Total Storage (kg) } \\
\hline Anderson & $1.37 \times 10^{10}(26)$ & $1.31 \times 10^{9}$ & 0 & 0 & 0 & 0 & 0 & $1.23 \times 10^{10}$ \\
\hline Cherokee & $8.98 \times 10^{9}(42)$ & $1.51 \times 10^{9}$ & $1.32 \times 10^{9}$ & 0 & $1.18 \times 10^{9}$ & 0 & 0 & $4.97 \times 10^{9}$ \\
\hline Greenville & $1.54 \times 10^{10}(20)$ & $1.38 \times 10^{9}$ & $2.32 \times 10^{9}$ & 0 & 0 & 0 & 0 & $1.17 \times 10^{10}$ \\
\hline Oconee & $1.21 \times 10^{10}(31)$ & $4.74 \times 10^{8}$ & $7.84 \times 10^{8}$ & 0 & 0 & 0 & 0 & $1.08 \times 10^{10}$ \\
\hline Pickens & $9.53 \times 10^{9}(39)$ & $4.99 \times 10^{8}$ & $7.70 \times 10^{8}$ & 0 & 0 & 0 & 0 & $8.26 \times 10^{9}$ \\
\hline Spartanburg & $1.49 \times 10^{10}(22)$ & $1.02 \times 10^{8}$ & $2.25 \times 10^{9}$ & 0 & $9.09 \times 10^{8}$ & 0 & 0 & $1.16 \times 10^{10}$ \\
\hline Union & $1.19 \times 10^{10}(32)$ & $9.47 \times 10^{8}$ & $8.54 \times 10^{8}$ & 0 & $4.30 \times 10^{9}$ & 0 & 0 & $5.84 \times 10^{9}$ \\
\hline (Upstate) & $8.64 \times 10^{10}(4)$ & $6.22 \times 10^{9}$ & $8.30 \times 10^{9}$ & 0 & $6.38 \times 10^{9}$ & 0 & 0 & $6.55 \times 10^{10}$ \\
\hline Abbeville & $1.15 \times 10^{10}(34)$ & $6.66 \times 10^{8}$ & $5.32 \times 10^{8}$ & 0 & $4.73 \times 10^{9}$ & 0 & 0 & $5.52 \times 10^{9}$ \\
\hline Aiken & $2.51 \times 10^{10}(7)$ & $5.70 \times 10^{9}$ & $2.98 \times 10^{9}$ & $1.57 \times 10^{9}$ & $5.90 \times 10^{7}$ & 0 & 0 & $1.48 \times 10^{10}$ \\
\hline Chester & $1.43 \times 10^{10}(24)$ & $7.68 \times 10^{8}$ & $1.15 \times 10^{9}$ & 0 & $6.73 \times 10^{9}$ & $1.50 \times 10^{8}$ & 0 & $5.50 \times 10^{9}$ \\
\hline Edgefield & $1.04 \times 10^{10}(37)$ & $1.29 \times 10^{9}$ & $7.84 \times 10^{8}$ & 0 & $7.20 \times 10^{8}$ & 0 & 0 & $7.60 \times 10^{9}$ \\
\hline Fairfield & $1.59 \times 10^{10}(17)$ & $1.28 \times 10^{7}$ & $2.45 \times 10^{9}$ & 0 & $6.90 \times 10^{9}$ & 0 & 0 & $6.55 \times 10^{9}$ \\
\hline Greenwood & $1.07 \times 10^{10}(36)$ & 0 & $1.62 \times 10^{9}$ & 0 & $3.95 \times 10^{9}$ & 0 & 0 & $5.11 \times 10^{9}$ \\
\hline Kershaw & $1.95 \times 10^{10}(11)$ & $4.90 \times 10^{9}$ & $2.25 \times 10^{9}$ & $2.85 \times 10^{9}$ & $5.55 \times 10^{8}$ & 0 & 0 & $8.95 \times 10^{9}$ \\
\hline Lancaster & $1.16 \times 10^{10}(33)$ & $6.78 \times 10^{8}$ & $2.11 \times 10^{9}$ & 0 & $7.32 \times 10^{8}$ & 0 & 0 & $8.12 \times 10^{9}$ \\
\hline Laurens & $1.55 \times 10^{10}(19)$ & $1.60 \times 10^{9}$ & $2.66 \times 10^{8}$ & 0 & $4.14 \times 10^{9}$ & 0 & 0 & $9.53 \times 10^{9}$ \\
\hline Lexington & $1.59 \times 10^{10}(18)$ & $5.81 \times 10^{9}$ & $1.20 \times 10^{9}$ & 0 & $4.25 \times 10^{8}$ & 0 & $1.29 \times 10^{8}$ & $8.31 \times 10^{9}$ \\
\hline Newberry & $1.37 \times 10^{10}(25)$ & $8.96 \times 10^{8}$ & $8.96 \times 10^{8}$ & 0 & $3.28 \times 10^{9}$ & 0 & 0 & $8.58 \times 10^{9}$ \\
\hline Richland & $1.76 \times 10^{10}(13)$ & $2.09 \times 10^{9}$ & $5.04 \times 10^{9}$ & $1.14 \times 10^{9}$ & $2.24 \times 10^{8}$ & 0 & 0 & $9.07 \times 10^{9}$ \\
\hline Saluda & $9.35 \times 10^{9}(40)$ & $2.43 \times 10^{8}$ & $1.16 \times 10^{9}$ & 0 & $9.09 \times 10^{8}$ & 0 & 0 & $7.04 \times 10^{9}$ \\
\hline York & $1.61 \times 10^{10}(16)$ & $6.40 \times 10^{7}$ & $1.88 \times 10^{9}$ & 0 & $6.81 \times 10^{9}$ & 0 & 0 & $7.36 \times 10^{9}$ \\
\hline (Midlands) & $2.06 \times 10^{11}(3)$ & $2.47 \times 10^{10}$ & $2.43 \times 10^{10}$ & $4.42 \times 10^{9}$ & $4.02 \times 10^{10}$ & $1.50 \times 10^{8}$ & $1.29 \times 10^{8}$ & $1.12 \times 10^{11}$ \\
\hline Chesterfield & $2.02 \times 10^{10}(10)$ & $2.21 \times 10^{9}$ & $9.17 \times 10^{9}$ & 0 & $2.71 \times 10^{8}$ & 0 & 0 & $8.53 \times 10^{9}$ \\
\hline Clarendon & $1.35 \times 10^{10}(28)$ & $4.99 \times 10^{8}$ & $2.69 \times 10^{9}$ & $8.55 \times 10^{8}$ & 0 & 0 & 0 & $9.44 \times 10^{9}$ \\
\hline Darlington & $1.34 \times 10^{10}(29)$ & $4.61 \times 10^{8}$ & $3.61 \times 10^{9}$ & $1.28 \times 10^{9}$ & 0 & 0 & $1.29 \times 10^{7}$ & $8.08 \times 10^{9}$ \\
\hline Dillon & $9.98 \times 10^{9}(38)$ & $1.16 \times 10^{9}$ & $1.79 \times 10^{9}$ & $1.14 \times 10^{9}$ & 0 & 0 & $2.45 \times 10^{8}$ & $5.64 \times 10^{9}$ \\
\hline Florence & $1.66 \times 10^{10}(15)$ & $1.24 \times 10^{9}$ & $3.14 \times 10^{9}$ & 0 & 0 & 0 & $2.58 \times 10^{7}$ & $1.22 \times 10^{10}$ \\
\hline Georgetown & $2.89 \times 10^{10}(3)$ & $4.49 \times 10^{9}$ & $3.84 \times 10^{9}$ & $8.12 \times 10^{9}$ & $4.83 \times 10^{9}$ & 0 & $1.48 \times 10^{9}$ & $6.09 \times 10^{9}$ \\
\hline Horry & $3.68 \times 10^{10}(1)$ & $3.23 \times 10^{9}$ & $6.03 \times 10^{9}$ & $9.12 \times 10^{9}$ & $3.39 \times 10^{9}$ & 0 & $4.26 \times 10^{9}$ & $1.08 \times 10^{10}$ \\
\hline Lee & $8.59 \times 10^{9}(43)$ & $3.71 \times 10^{8}$ & $1.83 \times 10^{9}$ & 0 & $1.18 \times 10^{7}$ & 0 & 0 & $6.37 \times 10^{9}$ \\
\hline Marion & $1.53 \times 10^{10}(21)$ & $1.37 \times 10^{9}$ & $4.00 \times 10^{9}$ & $3.85 \times 10^{9}$ & 0 & 0 & $6.32 \times 10^{8}$ & $5.48 \times 10^{9}$ \\
\hline Marlboro & $2.21 \times 10^{10}(8)$ & $9.60 \times 10^{8}$ & $3.77 \times 10^{9}$ & $1.15 \times 10^{10}$ & $2.01 \times 10^{8}$ & 0 & $2.58 \times 10^{7}$ & $5.58 \times 10^{9}$ \\
\hline Sumter & $1.45 \times 10^{10}(23)$ & $1.15 \times 10^{8}$ & $4.90 \times 10^{9}$ & 0 & $4.72 \times 10^{7}$ & 0 & $2.58 \times 10^{7}$ & $9.44 \times 10^{9}$ \\
\hline Williamsburg & $1.86 \times 10^{10}(12)$ & $3.20 \times 10^{8}$ & $2.93 \times 10^{9}$ & 0 & 0 & 0 & $3.87 \times 10^{7}$ & $1.54 \times 10^{10}$ \\
\hline (Pee Dee) & $2.19 \times 10^{11}(2)$ & $1.64 \times 10^{10}$ & $4.77 \times 10^{10}$ & $3.59 \times 10^{10}$ & $8.74 \times 10^{9}$ & 0 & $6.75 \times 10^{9}$ & $1.03 \times 10^{11}$ \\
\hline Allendale & $9.14 \times 10^{9}(41)$ & $3.20 \times 10^{8}$ & $1.41 \times 10^{9}$ & $8.55 \times 10^{8}$ & 0 & 0 & 0 & $6.55 \times 10^{9}$ \\
\hline Bamberg & $8.16 \times 10^{9}(45)$ & $1.61 \times 10^{9}$ & $1.40 \times 10^{7}$ & 0 & $4.84 \times 10^{8}$ & 0 & $3.87 \times 10^{7}$ & $6.01 \times 10^{9}$ \\
\hline Barnwell & $1.15 \times 10^{10}(35)$ & $9.98 \times 10^{8}$ & $1.93 \times 10^{9}$ & 0 & 0 & 0 & 0 & $8.52 \times 10^{9}$ \\
\hline Beaufort & $1.68 \times 10^{10}(14)$ & $8.93 \times 10^{9}$ & $4.76 \times 10^{8}$ & $8.55 \times 10^{8}$ & $4.72 \times 10^{8}$ & $5.75 \times 10^{8}$ & $2.71 \times 10^{9}$ & $2.78 \times 10^{9}$ \\
\hline Berkeley & $2.80 \times 10^{10}(5)$ & $1.86 \times 10^{9}$ & $2.91 \times 10^{9}$ & $3.28 \times 10^{9}$ & $4.83 \times 10^{9}$ & 0 & $1.77 \times 10^{9}$ & $1.34 \times 10^{10}$ \\
\hline Calhoun & $5.87 \times 10^{9}(46)$ & $8.45 \times 10^{8}$ & $3.64 \times 10^{8}$ & 0 & 0 & 0 & 0 & $4.66 \times 10^{9}$ \\
\hline Charleston & $2.81 \times 10^{10}(4)$ & $9.79 \times 10^{9}$ & $4.65 \times 10^{9}$ & 0 & $8.58 \times 10^{9}$ & 0 & $3.52 \times 10^{9}$ & $1.56 \times 10^{9}$ \\
\hline Colleton & $3.57 \times 10^{10}(2)$ & $3.58 \times 10^{9}$ & $6.86 \times 10^{8}$ & $1.25 \times 10^{10}$ & $1.65 \times 10^{9}$ & $2.13 \times 10^{9}$ & $1.41 \times 10^{9}$ & $1.37 \times 10^{10}$ \\
\hline Dorchester & $1.36 \times 10^{10}(27)$ & $3.51 \times 10^{9}$ & $2.38 \times 10^{8}$ & $1.43 \times 10^{8}$ & $3.30 \times 10^{9}$ & 0 & $3.87 \times 10^{8}$ & $6.06 \times 10^{9}$ \\
\hline Hampton & $1.27 \times 10^{10}(30)$ & $1.74 \times 10^{9}$ & $1.22 \times 10^{9}$ & $4.28 \times 10^{8}$ & $1.19 \times 10^{9}$ & 0 & $5.16 \times 10^{8}$ & $7.64 \times 10^{9}$ \\
\hline Jasper & $2.52 \times 10^{10}(6)$ & $4.07 \times 10^{9}$ & $8.12 \times 10^{8}$ & $8.12 \times 10^{9}$ & $2.90 \times 10^{9}$ & $2.90 \times 10^{9}$ & $3.10 \times 10^{8}$ & $6.04 \times 10^{9}$ \\
\hline McCormick & $8.51 \times 10^{9}(44)$ & $8.19 \times 10^{8}$ & $9.10 \times 10^{8}$ & 0 & $2.91 \times 10^{9}$ & 0 & 0 & $3.87 \times 10^{9}$ \\
\hline Orangeburg & $2.09 \times 10^{10}(9)$ & $9.34 \times 10^{8}$ & $2.52 \times 10^{8}$ & 0 & $2.95 \times 10^{8}$ & 0 & $1.29 \times 10^{7}$ & $1.94 \times 10^{10}$ \\
\hline (Low Country) & $2.24 \times 10^{11}(1)$ & $3.90 \times 10^{10}$ & $1.59 \times 10^{10}$ & $2.62 \times 10^{10}$ & $2.66 \times 10^{10}$ & $5.60 \times 10^{9}$ & $1.07 \times 10^{10}$ & $1.00 \times 10^{11}$ \\
\hline Totals (kg) & $7.36 \times 10^{11}$ & $8.64 \times 10^{10}$ & $9.62 \times 10^{10}$ & $6.77 \times 10^{10}$ & $8.19 \times 10^{10}$ & $5.75 \times 10^{9}$ & $1.75 \times 10^{10}$ & $3.81 \times 10^{11}$ \\
\hline
\end{tabular}


Table 10. Total soil carbon (TSC) values by county, region, and soil order for the state of South Carolina (U.S.A.), based on mid-point total soil carbon (TSC) numbers for the upper $2 \mathrm{~m}$ from Guo et al. 2006 [18] and a social cost of carbon (SC-CO 2 ) of $\$ 46$ per metric ton of $\mathrm{CO}_{2}$ (2007 U.S. dollars with an average discount rate of $3 \%$ [17]).

\begin{tabular}{|c|c|c|c|c|c|c|c|c|}
\hline \multirow{4}{*}{ County (Region) } & \multirow{4}{*}{$\begin{array}{c}\text { Total Value } \\
\text { (\$) } \\
\text { (Rank) }\end{array}$} & \multicolumn{7}{|c|}{ Slight $<\longrightarrow$ Degree of Weathering and Soil Development $\longrightarrow$ Strong } \\
\hline & & \multicolumn{3}{|c|}{ Slightly Weathered } & \multicolumn{2}{|c|}{ Moderately Weathered } & \multicolumn{2}{|c|}{ Strongly Weathered } \\
\hline & & Entisols & Inceptisols & Histosols & Alfisols & Mollisols & Spodosols & Ultisols \\
\hline & & \multicolumn{7}{|c|}{ Value (\$) } \\
\hline Anderson & $2.31 \times 10^{9}(26)$ & $2.21 \times 10^{8}$ & 0 & 0 & 0 & 0 & 0 & $2.09 \times 10^{9}$ \\
\hline Cherokee & $1.52 \times 10^{9}(42)$ & $2.56 \times 10^{8}$ & $2.22 \times 10^{8}$ & 0 & $1.99 \times 10^{8}$ & 0 & 0 & $8.40 \times 10^{8}$ \\
\hline Greenville & $2.60 \times 10^{9}(20)$ & $2.34 \times 10^{8}$ & $3.92 \times 10^{8}$ & 0 & 0 & 0 & 0 & $1.97 \times 10^{9}$ \\
\hline Oconee & $2.04 \times 10^{9}(31)$ & $8.03 \times 10^{7}$ & $1.32 \times 10^{8}$ & 0 & 0 & 0 & 0 & $1.83 \times 10^{9}$ \\
\hline Pickens & $1.61 \times 10^{9}(39)$ & $8.46 \times 10^{7}$ & $1.30 \times 10^{8}$ & 0 & 0 & 0 & 0 & $1.40 \times 10^{9}$ \\
\hline Spartanburg & $2.51 \times 10^{9}(22)$ & $1.74 \times 10^{7}$ & $3.80 \times 10^{8}$ & 0 & $1.53 \times 10^{8}$ & 0 & 0 & $1.96 \times 10^{9}$ \\
\hline Union & $2.02 \times 10^{9}(32)$ & $1.61 \times 10^{8}$ & $1.44 \times 10^{8}$ & 0 & $7.24 \times 10^{8}$ & 0 & 0 & $9.88 \times 10^{8}$ \\
\hline (Upstate) & $1.46 \times 10^{10}(4)$ & $1.05 \times 10^{9}$ & $1.40 \times 10^{9}$ & 0 & $1.08 \times 10^{9}$ & 0 & 0 & $1.11 \times 10^{10}$ \\
\hline Abbeville & $1.93 \times 10^{9}(34)$ & $1.13 \times 10^{8}$ & $8.97 \times 10^{7}$ & 0 & $7.98 \times 10^{8}$ & 0 & 0 & $9.34 \times 10^{8}$ \\
\hline Aiken & $4.24 \times 10^{9}(7)$ & $9.66 \times 10^{8}$ & $5.03 \times 10^{8}$ & $2.64 \times 10^{8}$ & $9.95 \times 10^{6}$ & 0 & 0 & $2.50 \times 10^{9}$ \\
\hline Chester & $2.41 \times 10^{9}(24)$ & $1.30 \times 10^{8}$ & $1.94 \times 10^{8}$ & 0 & $1.13 \times 10^{9}$ & $2.53 \times 10^{7}$ & 0 & $9.30 \times 10^{8}$ \\
\hline Edgefield & $1.76 \times 10^{9}(37)$ & $2.19 \times 10^{8}$ & $1.32 \times 10^{8}$ & 0 & $1.21 \times 10^{8}$ & 0 & 0 & $1.29 \times 10^{9}$ \\
\hline Fairfield & $2.69 \times 10^{9}(17)$ & $2.17 \times 10^{6}$ & $4.13 \times 10^{8}$ & 0 & $1.16 \times 10^{9}$ & 0 & 0 & $1.11 \times 10^{9}$ \\
\hline Greenwood & $1.80 \times 10^{9}(36)$ & 0 & $2.74 \times 10^{8}$ & 0 & $6.67 \times 10^{8}$ & 0 & 0 & $8.64 \times 10^{8}$ \\
\hline Kershaw & $3.30 \times 10^{9}(11)$ & $8.31 \times 10^{8}$ & $3.80 \times 10^{8}$ & $4.81 \times 10^{8}$ & $9.35 \times 10^{7}$ & 0 & 0 & $1.51 \times 10^{9}$ \\
\hline Lancaster & $1.97 \times 10^{9}(33)$ & $1.15 \times 10^{8}$ & $3.56 \times 10^{8}$ & 0 & $1.23 \times 10^{8}$ & 0 & 0 & $1.37 \times 10^{9}$ \\
\hline Laurens & $2.62 \times 10^{9}(19)$ & $2.71 \times 10^{8}$ & $4.48 \times 10^{7}$ & 0 & $6.98 \times 10^{8}$ & 0 & 0 & $1.61 \times 10^{9}$ \\
\hline Lexington & $2.69 \times 10^{9}(18)$ & $9.85 \times 10^{8}$ & $2.03 \times 10^{8}$ & 0 & $7.16 \times 10^{7}$ & 0 & $2.17 \times 10^{7}$ & $1.40 \times 10^{9}$ \\
\hline Newberry & $2.31 \times 10^{9}(25)$ & $1.52 \times 10^{8}$ & $1.51 \times 10^{8}$ & 0 & $5.53 \times 10^{8}$ & 0 & 0 & $1.45 \times 10^{9}$ \\
\hline Richland & $2.97 \times 10^{9}(13)$ & $3.54 \times 10^{8}$ & $8.50 \times 10^{8}$ & $1.92 \times 10^{8}$ & $3.78 \times 10^{7}$ & 0 & 0 & $1.53 \times 10^{9}$ \\
\hline Saluda & $1.58 \times 10^{9}(40)$ & $4.12 \times 10^{7}$ & $1.96 \times 10^{8}$ & 0 & $1.53 \times 10^{8}$ & 0 & 0 & $1.19 \times 10^{9}$ \\
\hline York & $2.72 \times 10^{9}(16)$ & $1.09 \times 10^{7}$ & $3.16 \times 10^{8}$ & 0 & $1.15 \times 10^{9}$ & 0 & 0 & $1.24 \times 10^{9}$ \\
\hline (Midlands) & $3.48 \times 10^{10}(3)$ & $4.19 \times 10^{9}$ & $4.10 \times 10^{9}$ & $7.45 \times 10^{8}$ & $6.77 \times 10^{9}$ & $2.53 \times 10^{7}$ & $2.17 \times 10^{7}$ & $1.89 \times 10^{10}$ \\
\hline Chesterfield & $3.41 \times 10^{9}(10)$ & $3.75 \times 10^{8}$ & $1.55 \times 10^{9}$ & 0 & $4.58 \times 10^{7}$ & 0 & 0 & $1.44 \times 10^{9}$ \\
\hline Clarendon & $2.28 \times 10^{9}(28)$ & $8.46 \times 10^{7}$ & $4.53 \times 10^{8}$ & $1.44 \times 10^{8}$ & 0 & 0 & 0 & $1.59 \times 10^{9}$ \\
\hline Darlington & $2.27 \times 10^{9}(29)$ & $7.81 \times 10^{7}$ & $6.09 \times 10^{8}$ & $2.16 \times 10^{8}$ & 0 & 0 & $2.17 \times 10^{6}$ & $1.37 \times 10^{9}$ \\
\hline Dillon & $1.69 \times 10^{9}(38)$ & $1.97 \times 10^{8}$ & $3.02 \times 10^{8}$ & $1.92 \times 10^{8}$ & 0 & 0 & $4.12 \times 10^{7}$ & $9.53 \times 10^{8}$ \\
\hline Florence & $2.81 \times 10^{9}(15)$ & $2.10 \times 10^{8}$ & $5.29 \times 10^{8}$ & 0 & 0 & 0 & $4.34 \times 10^{6}$ & $2.07 \times 10^{9}$ \\
\hline Georgetown & $4.87 \times 10^{9}(3)$ & $7.62 \times 10^{8}$ & $6.47 \times 10^{8}$ & $1.37 \times 10^{9}$ & $8.14 \times 10^{8}$ & 0 & $2.50 \times 10^{8}$ & $1.03 \times 10^{9}$ \\
\hline Horry & $6.22 \times 10^{9}(1)$ & $5.47 \times 10^{8}$ & $1.02 \times 10^{9}$ & $1.54 \times 10^{9}$ & $5.71 \times 10^{8}$ & 0 & $7.16 \times 10^{8}$ & $1.83 \times 10^{9}$ \\
\hline Lee & $1.45 \times 10^{9}(43)$ & $6.29 \times 10^{7}$ & $3.09 \times 10^{8}$ & 0 & $1.99 \times 10^{6}$ & 0 & 0 & $1.08 \times 10^{9}$ \\
\hline Marion & $2.59 \times 10^{9}(21)$ & $2.32 \times 10^{8}$ & $6.75 \times 10^{8}$ & $6.49 \times 10^{8}$ & 0 & 0 & $1.06 \times 10^{8}$ & $9.26 \times 10^{8}$ \\
\hline Marlboro & $3.73 \times 10^{9}(8)$ & $1.63 \times 10^{8}$ & $6.35 \times 10^{8}$ & $1.95 \times 10^{9}$ & $3.38 \times 10^{7}$ & 0 & $4.34 \times 10^{6}$ & $9.43 \times 10^{8}$ \\
\hline Sumter & $2.45 \times 10^{9}(23)$ & $1.95 \times 10^{7}$ & $8.26 \times 10^{8}$ & 0 & $7.96 \times 10^{6}$ & 0 & $4.34 \times 10^{6}$ & $1.59 \times 10^{9}$ \\
\hline Williamsburg & $3.15 \times 10^{9}(12)$ & $5.43 \times 10^{7}$ & $4.93 \times 10^{8}$ & 0 & 0 & 0 & $6.51 \times 10^{6}$ & $2.60 \times 10^{9}$ \\
\hline (Pee Dee) & $3.69 \times 10^{10}(2)$ & $2.79 \times 10^{9}$ & $8.04 \times 10^{9}$ & $6.06 \times 10^{9}$ & $1.47 \times 10^{9}$ & 0 & $1.13 \times 10^{9}$ & $1.74 \times 10^{10}$ \\
\hline Allendale & $1.54 \times 10^{9}(41)$ & $5.43 \times 10^{7}$ & $2.38 \times 10^{8}$ & $1.44 \times 10^{8}$ & 0 & 0 & 0 & $1.11 \times 10^{9}$ \\
\hline Bamberg & $1.38 \times 10^{9}(45)$ & $2.73 \times 10^{8}$ & $2.36 \times 10^{6}$ & 0 & $8.16 \times 10^{7}$ & 0 & $6.51 \times 10^{6}$ & $1.02 \times 10^{9}$ \\
\hline Barnwell & $1.93 \times 10^{9}(35)$ & $1.69 \times 10^{8}$ & $3.26 \times 10^{8}$ & 0 & 0 & 0 & 0 & $1.44 \times 10^{9}$ \\
\hline Beaufort & $2.84 \times 10^{9}(14)$ & $1.51 \times 10^{9}$ & $8.02 \times 10^{7}$ & $1.44 \times 10^{8}$ & $7.96 \times 10^{7}$ & $9.68 \times 10^{7}$ & $4.56 \times 10^{8}$ & $4.69 \times 10^{8}$ \\
\hline Berkeley & $4.73 \times 10^{9}(5)$ & $3.15 \times 10^{8}$ & $4.91 \times 10^{8}$ & $5.53 \times 10^{8}$ & $8.14 \times 10^{8}$ & 0 & $2.97 \times 10^{8}$ & $2.26 \times 10^{9}$ \\
\hline Calhoun & $9.92 \times 10^{8}(46)$ & $1.43 \times 10^{8}$ & $6.14 \times 10^{7}$ & 0 & 0 & 0 & 0 & $7.87 \times 10^{8}$ \\
\hline Charleston & $4.75 \times 10^{9}(4)$ & $1.66 \times 10^{9}$ & $7.84 \times 10^{8}$ & 0 & $1.45 \times 10^{9}$ & 0 & $5.92 \times 10^{8}$ & $2.64 \times 10^{8}$ \\
\hline Colleton & $6.02 \times 10^{9}(2)$ & $6.08 \times 10^{8}$ & $1.16 \times 10^{8}$ & $2.11 \times 10^{9}$ & $2.79 \times 10^{8}$ & $3.58 \times 10^{8}$ & $2.37 \times 10^{8}$ & $2.31 \times 10^{9}$ \\
\hline Dorchester & $2.30 \times 10^{9}(27)$ & $5.95 \times 10^{8}$ & $4.01 \times 10^{7}$ & $2.40 \times 10^{7}$ & $5.57 \times 10^{8}$ & 0 & $6.51 \times 10^{7}$ & $1.02 \times 10^{9}$ \\
\hline Hampton & $2.15 \times 10^{9}(30)$ & $2.95 \times 10^{8}$ & $2.05 \times 10^{8}$ & $7.21 \times 10^{7}$ & $2.01 \times 10^{8}$ & 0 & $8.68 \times 10^{7}$ & $1.29 \times 10^{9}$ \\
\hline Jasper & $4.25 \times 10^{9}(6)$ & $6.90 \times 10^{8}$ & $1.37 \times 10^{8}$ & $1.37 \times 10^{9}$ & $4.90 \times 10^{8}$ & $4.88 \times 10^{8}$ & $5.21 \times 10^{7}$ & $1.02 \times 10^{9}$ \\
\hline McCormick & $1.44 \times 10^{9}(44)$ & $1.39 \times 10^{8}$ & $1.53 \times 10^{8}$ & 0 & $4.92 \times 10^{8}$ & 0 & 0 & $6.54 \times 10^{8}$ \\
\hline Orangeburg & $3.53 \times 10^{9}(9)$ & $1.58 \times 10^{8}$ & $4.25 \times 10^{7}$ & 0 & $4.98 \times 10^{7}$ & 0 & $2.17 \times 10^{6}$ & $3.27 \times 10^{9}$ \\
\hline (Low Country) & $3.79 \times 10^{10}(1)$ & $6.61 \times 10^{9}$ & $2.68 \times 10^{9}$ & $4.42 \times 10^{9}$ & $4.49 \times 10^{9}$ & $9.43 \times 10^{8}$ & $1.79 \times 10^{9}$ & $1.69 \times 10^{10}$ \\
\hline Totals (\$) & $1.24 \times 10^{11}$ & $1.46 \times 10^{10}$ & $1.62 \times 10^{10}$ & $1.14 \times 10^{10}$ & $1.38 \times 10^{10}$ & $9.68 \times 10^{8}$ & $2.95 \times 10^{9}$ & $6.43 \times 10^{10}$ \\
\hline
\end{tabular}




\section{Discussion}

Pedodiversity (soil diversity) in South Carolina is a source of various ES goods, services, and disservices (ED). This study demonstrates the value of regulating ES/ED in the state and its regions and counties. According to Mikhailova et al. (2021) [22], taxonomic pedodiversity (e.g., soil order) "provides a general description of the stock, its type, and spatial distribution," which is often rereferred to as a "portfolio" to describe the link between pedodiversity and its stocks. South Carolina soil "portfolio" is composed of seven soil orders: Entisols (9\% of the total state area), Inceptisols (9\%), Histosols (1\%), Alfisols (9\%), Mollisols ( $0 \%$ ), Spodosols ( $2 \%$ ), and Ultisols (70\%) (Figure 4, Table 11). Highly weathered Ultisols have the highest proportion of the total area of the state (Figure 4a), which contributes to the highest SOC and TSC storage and their associated social costs of carbon. The contribution of SIC to associated social costs of carbon is small at the state level and primarily associated with Inceptisols, Entisols, and Alfisols.
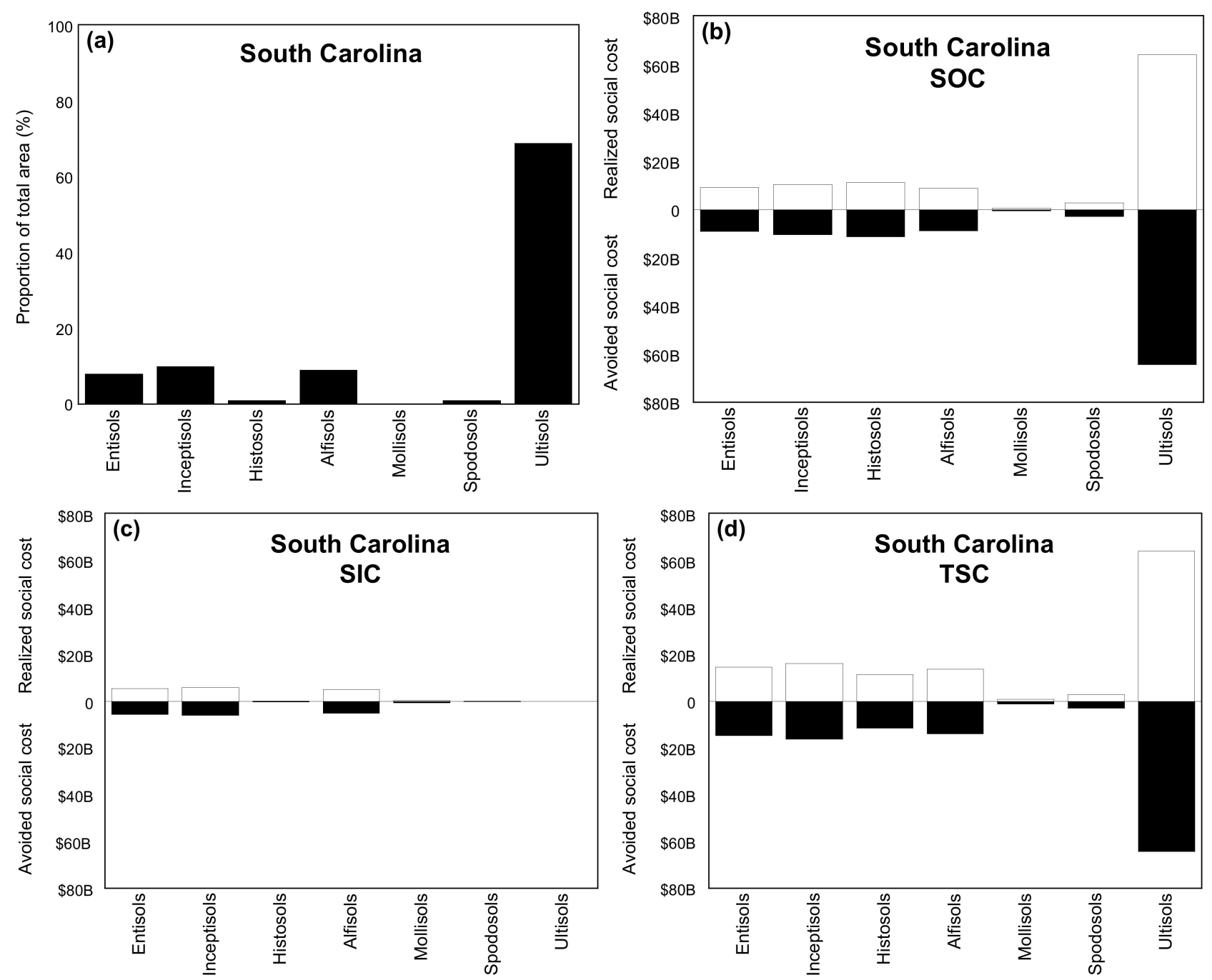

Figure 4. Diagram showing how the "portfolio-effect" and "distribution-effect" of pedodiversity can vary within the state: (a) pedodiversity by soil order area; (b) value of soil organic carbon (SOC) storage, (c) value of soil inorganic carbon (SIC) storage, (d) value of total soil carbon (TSC) storage in the upper 2-m depth based on avoided or realized the social cost of $\mathrm{CO}_{2}\left(\mathrm{SC}-\mathrm{CO}_{2}\right)$ of $\$ 46$ (USD) per metric ton of $\mathrm{CO}_{2}$ [17] by soil order. Note: $\mathrm{B}=$ billion $=10^{9}$.

Soil "portfolio" differs within each county, and Figure 5 illustrates this concept using three counties from different regions: Anderson (Upstate), Newberry (Midlands), and Colleton (Low Country). 

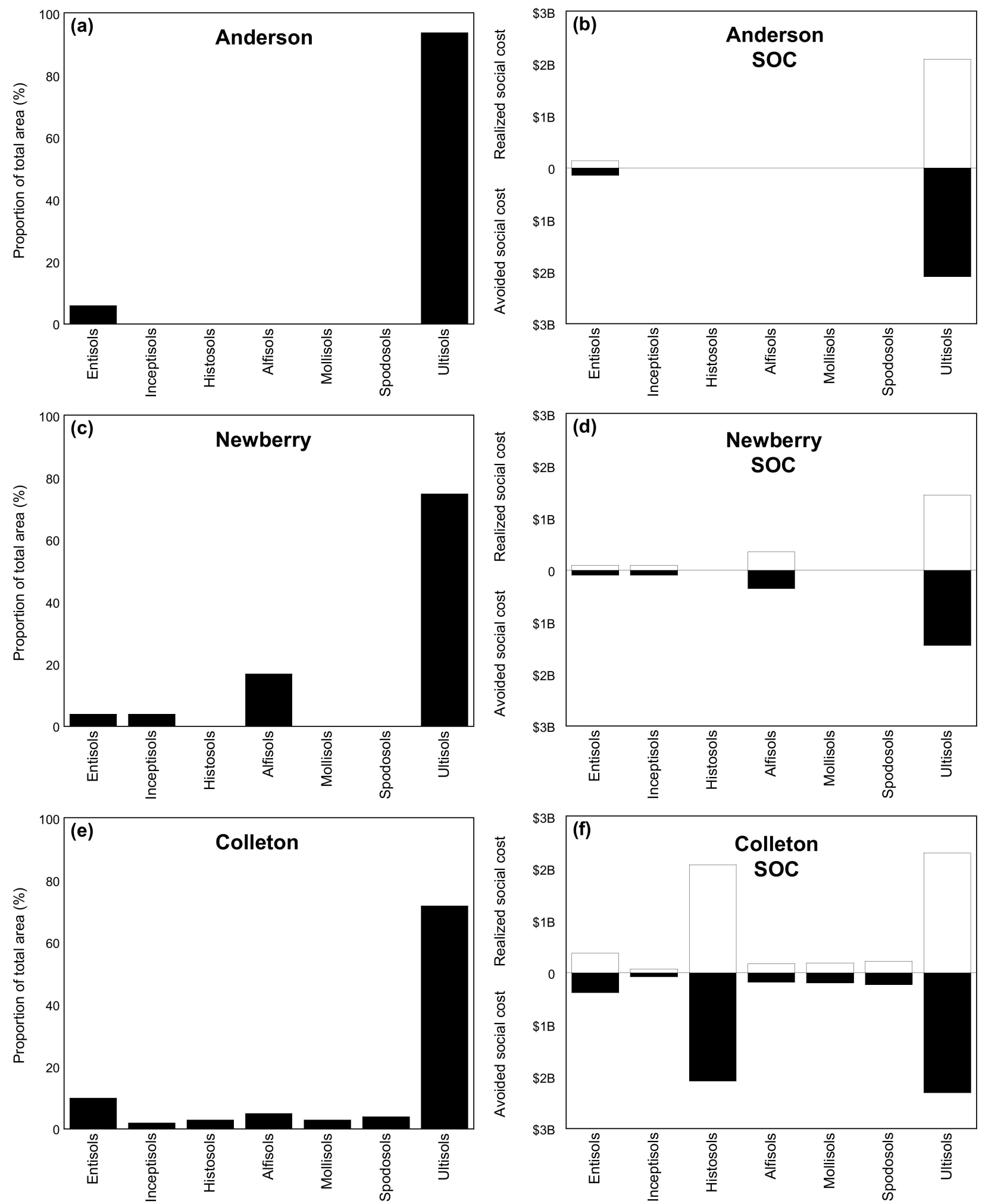

Figure 5. Diagram showing how the "portfolio-effect" and "distribution-effect" of pedodiversity can vary by county: (a,c,e) pedodiversity by soil order area; $(\mathbf{b}, \mathbf{d}, \mathbf{f})$ value of soil organic carbon (SOC) storage in the upper 2-m depth based on avoided or realized the social cost of $\mathrm{CO}_{2}\left(\mathrm{SC}-\mathrm{CO}_{2}\right)$ of $\$ 46$ (USD) per metric ton of $\mathrm{CO}_{2}$ [17] by soil order. Note: $\mathrm{B}=$ billion $=10^{9}$.

In all three cases, Ultisols occupy the largest proportion of the area in each county. The type of soil order influences the value of SOC storage. In Colleton County, the soil order of Histosols contributes to the social costs of $\mathrm{C}$ as much as the Ultisols even though its area is much smaller (Figure 5) because of high SOC content of $142.5 \mathrm{~kg} \mathrm{~m}^{-2}$. Figures 4 and 5 
represent social costs of soil C from different point of views: "avoided" versus "realized" social costs. Soil carbon stored in the soil represents the "avoided social cost" of soil C if not converted to $\mathrm{CO}_{2}$ and released into the atmosphere. When $\mathrm{CO}_{2}$ is released into the atmosphere, it becomes the "realized social cost" because of the damages from global warming. In South Carolina, Histosols and Alfisols are particularly sensitive to climate change because of relatively high soil $\mathrm{C}$ content, which is most likely to experience higher decomposition rates due to increases in temperature and precipitation. All soils in the state of South Carolina have low recarbonization potential.

Table 11. Distribution of soil carbon regulating ecosystem services in the state of South Carolina (U.S.A.) by soil order (photos courtesy of USDA/NRCS [23]) in the upper 2-m depth based on avoided or realized the social $\operatorname{cost}$ of $\mathrm{CO}_{2}\left(\mathrm{SC}^{\left.-\mathrm{CO}_{2}\right)}\right.$ of $\$ 46$ (USD) per metric ton of $\mathrm{CO}_{2}$ [17].

\begin{tabular}{|c|c|c|c|c|c|c|}
\hline \multicolumn{7}{|c|}{ Soil Regulating Ecosystem Services in the State of South Carolina } \\
\hline \multicolumn{7}{|c|}{ Slight $<\longrightarrow$ Degree of Weathering and Soil Development $\longrightarrow$ Strong } \\
\hline \multicolumn{3}{|c|}{$\begin{array}{c}\text { Slightly Weathered } \\
18 \%\end{array}$} & \multicolumn{2}{|c|}{$\begin{array}{c}\text { Moderately Weathered } \\
\mathbf{9 \%}\end{array}$} & \multicolumn{2}{|c|}{$\begin{array}{c}\text { Strongly Weathered } \\
72 \%\end{array}$} \\
\hline $\begin{array}{c}\text { Entisols } \\
9 \%\end{array}$ & $\begin{array}{c}\text { Inceptisols } \\
9 \%\end{array}$ & $\begin{array}{c}\text { Histosols } \\
1 \%\end{array}$ & $\begin{array}{c}\text { Alfisols } \\
9 \%\end{array}$ & $\begin{array}{c}\text { Mollisols } \\
0 \%\end{array}$ & $\begin{array}{c}\text { Spodosols } \\
2 \%\end{array}$ & $\begin{array}{l}\text { Ultisols } \\
70 \%\end{array}$ \\
\hline 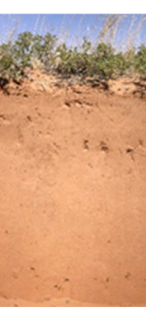 & & 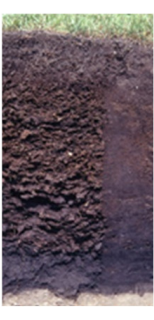 & 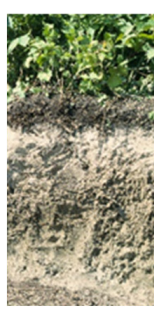 & & & \\
\hline \multicolumn{7}{|c|}{ The social cost of soil organic carbon (SOC) in USD: $\$ 107.14 B$} \\
\hline$\$ 9.11 \mathrm{~B}$ & $\$ 10.30 \mathrm{~B}$ & $\$ 11.20 \mathrm{~B}$ & $\$ 8.82 \mathrm{~B}$ & $\$ 524.00 \mathrm{M}$ & $\$ 2.82 \mathrm{~B}$ & $\$ 64.30 \mathrm{~B}$ \\
\hline $9 \%$ & $10 \%$ & $10 \%$ & $8 \%$ & $0 \%$ & $3 \%$ & $60 \%$ \\
\hline \multicolumn{7}{|c|}{ The social cost of soil inorganic carbon (SIC) in USD: $\$ 17.22 B$} \\
\hline$\$ 5.53 \mathrm{~B}$ & $\$ 5.91 B$ & $\$ 195.00 \mathrm{M}$ & $\$ 5.00 \mathrm{~B}$ & $\$ 444.00 \mathrm{M}$ & $\$ 136.00 \mathrm{M}$ & $\$ 0$ \\
\hline $32 \%$ & $34 \%$ & $1 \%$ & $29 \%$ & $3 \%$ & $1 \%$ & $0 \%$ \\
\hline \multicolumn{7}{|c|}{ The social cost of total soil carbon (TSC) in USD: $\$ 124.36 \mathrm{~B}$} \\
\hline$\$ 14.60 \mathrm{~B}$ & $\$ 16.20 \mathrm{~B}$ & $\$ 11.40 \mathrm{~B}$ & $\$ 13.80 \mathrm{~B}$ & $\$ 968.00 \mathrm{M}$ & $\$ 2.95 \mathrm{~B}$ & $\$ 64.30 \mathrm{~B}$ \\
\hline $12 \%$ & $13 \%$ & $9 \%$ & $11 \%$ & $1 \%$ & $2 \%$ & $52 \%$ \\
\hline \multicolumn{7}{|c|}{ Sensitivity to climate change } \\
\hline Low & Low & High & High & High & Low & Low \\
\hline \multicolumn{7}{|c|}{ Soil organic and inorganic carbon sequestration (recarbonization) potential } \\
\hline Low & Low & Low & Low & Low & Low & Low \\
\hline
\end{tabular}

Note: Entisols, Inceptisols, Alfisols, Mollisols, Spodosols, Ultisols are mineral soils. Histosols are mostly organic soils. $\mathrm{M}=\mathrm{million}=10^{6}$; $\mathrm{B}=$ billion $=10^{9}$.

Amelung et al. (2020) [24] proposed linking soil C sequestration to food security using soil- and site-specific potentials and opportunities for soil C sequestration. In this respect, the state of South Carolina faces serious limitations in both soil- (dominated by highlyweathered soil order, Ultisols) and site-specific (high demand for soil C due to rapid urbanization and population growth; rapid changes in coastal areas, etc.) potentials. Soil order Histosols (which often contains organic soils) is located in the coastal areas of the state and can be drained for agriculture and urbanization, leading to high losses of soil $\mathrm{C}$ into the atmosphere [24]. Recarbonization of soils in the state of South Carolina may 
not be economically feasible due to past excessive levels of soil degradation [25], high fertilization and liming costs (including transportation) associated with increasing soil $\mathrm{C}$ in mostly highly-weathered and acid soils in the state. It should be noted that the reported soil survey-based $\mathrm{C}$ values may be an overestimate of actual soil $\mathrm{C}$ measured in the field, but the overall trends for the soil orders should be similar [10]. Soil C should be regularly monitored to quantify soil contributions to ES and its flows [26,27].

\section{Conclusions}

This study examined the application of soil diversity (pedodiversity) concepts (taxonomic) and its measures to value soil C regulating ES/ED in the state of South Carolina (U.S.A.), its administrative units (regions, counties), and the systems of soil classification (e.g., U.S. Department of Agriculture (USDA) Soil Taxonomy, Soil Survey Geographic (SSURGO) Database) to be considered in territorial planning. Pedodiversity provides a critical context (e.g., "portfolio-effect," "distribution-effect," "evenness-effect," etc.) for analyzing, interpreting, and reporting ES/ED within the ES framework for sustainable management of soil carbon within the state. Taxonomic pedodiversity in South Carolina exhibits high soil diversity (7 soil orders: Entisols, Inceptisols, Histosols, Alfisols, Mollisols, Spodosols, and Ultisols), which is not evenly distributed within the state, regions, and counties. In general, pedodiversity tends to increase from the Upstate to Low Country, where three counties (Beaufort, Colleton, and Jasper) have all seven orders. Similarly, soil carbon storage and its associated social costs tend to increase in a similar geographic direction. Ultisols occupy the highest proportion of the state area (70\%) and have the highest SOC storage and related social costs of carbon $(\$ 64.30 \mathrm{~B})$. The contribution of SIC to associated social costs of carbon is small (\$17.22B) at the state level and primarily associated with Inceptisols (\$5.91B), Entisols (\$5.53B), and Alfisols (\$5.00B). In the state of South Carolina, Histosols and Alfisols are particularly sensitive to climate change because of relatively high soil C content, which is most likely experience higher rates of decomposition due to increases in temperature and precipitation. All soils in the state of South Carolina have low recarbonization potential. Administrative areas (e.g., counties, regions) combined with pedodiversity concepts can provide useful information to design cost-efficient policies to manage soil carbon regulating ES at the state level.

Author Contributions: Conceptualization, E.A.M.; methodology, E.A.M., M.A.S. and H.A.Z.; formal analysis, E.A.M.; writing—original draft preparation, E.A.M.; writing—review and editing, E.A.M., C.J.P., G.C.P. and M.A.S.; visualization, H.A.Z., L.L. and Z.H. All authors have read and agreed to the published version of the manuscript.

Funding: This research received no external funding.

Data Availability Statement: Not applicable.

Acknowledgments: We would like to thank the reviewers for their constructive comments and suggestions.

Conflicts of Interest: The authors declare no conflict of interest. 


$\begin{array}{ll}\text { Abbreviations } \\ \text { ED } & \text { Ecosystem disservices } \\ \text { ES } & \text { Ecosystem services } \\ \text { EPA } & \text { Environmental Protection Agency } \\ \text { SC-CO } 2 & \text { Social cost of carbon emissions } \\ \text { SDGs } & \text { Sustainable Development Goals } \\ \text { SOC } & \text { Soil organic carbon } \\ \text { SIC } & \text { Soil inorganic carbon } \\ \text { SOM } & \text { Soil organic matter } \\ \text { SSURGO } & \text { Soil Survey Geographic Database } \\ \text { TSC } & \text { Total soil carbon } \\ \text { USDA } & \text { United States Department of Agriculture } \\ \text { U.S.A. } & \text { United States of America }\end{array}$

\section{References}

1. Keestra, S.D.; Bouma, J.; Wallinga, J.; Tittonell, P.; Smith, P.; Cerda, A.; Montanarella, L.; Quinton, J.N.; Pachepsky, Y.; Van der Putten, W.H.; et al. The significance of soils and soil science towards realization of the United Nations Sustainable Development Goals. Soil 2016, 2, 111-128. [CrossRef]

2. Wood, S.L.; Jones, S.K.; Johnson, J.A.; Brauman, K.A.; Chaplin-Kramer, R.; Fremier, A.; Girvetz, E.; Gordon, L.J.; Kappel, C.V.; Mandle, L.; et al. Distilling the role of ecosystem services in the Sustainable Development Goals. Ecosyt. Serv. 2017, $29,701-782$. [CrossRef]

3. Adhikari, K.; Hartemink, A.E. Linking soils to ecosystem services-A global review. Geoderma 2016, 262, 101-111. [CrossRef]

4. Plaster, E.J. Soil Science and Management, 4th ed.; Delmar Learning, a Division of Thomson Learning, Inc.: Clifton Park, NY, USA, 2003; ISBN 0766839362.

5. Mikhailova, E.A.; Post, C.J.; Schlautman, M.A.; Post, G.C.; Zurqani, H.A. The business side of ecosystem services of soil systems. Earth 2020, 1, 15-34. [CrossRef]

6. Feller, C.; Manlay, R.J.; Swift, M.J.; Bernoux, M. Functions, services and value of soil organic matter for human societies and the environment: A historical perspective. In Functions of Soils for Human Societies and the Environment, 2nd ed.; Frossard, E., Blum, W.E.H., Warkentin, B.P., Eds.; Geological Society of London: London, UK, 2006; Volume 206, pp. 9-22.

7. Sheikh, M.A.; Kumar, M.; Todaria, N.P.; Pandey, R. Biomass and soil carbon along altitudinal gradients in temperate Cedrus deodara forests in Central Himalaya, India: Implications for climate change mitigation. Ecol. Indic. 2020, 111, 106025. [CrossRef]

8. Mikhailova, E.A.; Groshans, G.R.; Post, C.J.; Schlautman, M.A.; Post, G.C. Valuation of soil organic carbon stocks in the contiguous United States based on the avoided social cost of carbon emissions. Resources 2019, 8, 153. [CrossRef]

9. Groshans, G.R.; Mikhailova, E.A.; Post, C.J.; Schlautman, M.A.; Zhang, L. Determining the value of soil inorganic carbon stocks in the contiguous United States based on the avoided social cost of carbon emissions. Resources 2019, 8, 119. [CrossRef]

10. Mikhailova, E.A.; Post, C.J.; Schlautman, M.A.; Post, C.J.; Zurqani, H.A. Determining farm-scale site-specific monetary values of "soil carbon hotspots" based on avoided social costs of $\mathrm{CO}_{2}$ emissions. Cogent Environ. Sci. 2020, 6, 1-1817289. [CrossRef]

11. Mikhailova, E.A.; Groshans, G.R.; Post, C.J.; Schlautman, M.A.; Post, C.J. Valuation of total soil carbon stocks in the contiguous United States based on the avoided social cost of carbon emissions. Resources 2019, 8, 157. [CrossRef]

12. Lehmann, J.; Kleber, M. The contentious nature of soil organic matter. Nature 2015, 528, 60-68. [CrossRef] [PubMed]

13. Heaton, L.; Fullen, M.A.; Bhattacharyya, R. Critical analysis of the van Bemmelen conversion factor used to convert soil organic matter data to soil organic carbon data: Comparative analyses in a UK loamy sand soil. Espaço Aberto 2016, 6, 35-44. [CrossRef]

14. Mikhailova, E.A.; Goddard, M.A.; Post, C.J.; Schlautman, M.A.; Galbraith, J.M. Potential contribution of combined atmospheric $\mathrm{Ca}^{2+}$ and $\mathrm{Mg}^{2+}$ wet deposition within the continental U.S. to soil inorganic carbon sequestration. Pedosphere 2013, $23,808-814$. [CrossRef]

15. Soil Survey Staff, Natural Resources Conservation Service, United States Department of Agriculture. Soil Survey Geographic (SSURGO) Database. Available online: https://nrcs.app.box.com/v/soils (accessed on 10 September 2020).

16. Fossey, M.; Angers, D.; Bustany, C.; Cudennec, C.; Durand, P.; Gascuel-Odoux, C.; Jaffrezic, A.; Pérès, G.; Besse, C.; Walter, C. A framework to consider soil ecosystem services in territorial planning. Front. Environ. Sci. 2020, 8. Article 28. [CrossRef]

17. EPA. The Social Cost of Carbon. EPA Fact Sheet. 2016. Available online: https://19january2017snapshot.epa.gov/climatechange/ social-cost-carbon_.html (accessed on 15 March 2019).

18. Guo, Y.; Amundson, R.; Gong, P.; Yu, Q. Quantity and spatial variability of soil carbon in the conterminous United States. Soil Sci. Soc. Am. J. 2006, 70, 590-600. [CrossRef]

19. Groshans, G.R.; Mikhailova, E.A.; Post, C.J.; Schlautman, M.A. Accounting for soil inorganic carbon in the ecosystem services framework for the United Nations sustainable development goals. Geoderma 2018, 324, 37-46. [CrossRef]

20. The United States Census Bureau, 2018 TIGER/Line Boundary Shapefiles. Available online: https://www.census.gov/geographies/ mapping-files/time-series / geo/tiger-line-file.2018.html (accessed on 10 October 2020). 
21. Burt, R. Soil Survey Laboratory Methods Manual. Soil Survey Investigations Report No. 42 Version 3. U.S. Department of Agriculture, Natural Resources Conservation Service. 1996. Available online: https:/ / www.nrcs.usda.gov/Internet/FSE_DOCUMENTS/ nrcseprd1026806.pdf (accessed on 10 October 2020).

22. Mikhailova, E.A.; Zurqani, H.A.; Post, C.J.; Schlautman, M.A.; Post, C.J. Soil diversity (pedodiversity) and ecosystem services. Land 2021, 10, 288. [CrossRef]

23. Soil Survey Staff, Natural Resources Conservation Service, United States Department of Agriculture. Photos of soil orders. Available online: https://www.nrcs.usda.gov/wps/portal/nrcs/detail/soils/edu/?cid=nrcs142p2_053588 (accessed on 20 February 2021).

24. Amelung, W.; Bossio, D.; de Vries, W.; Kögel-Knabner, I.; Lehmann, J.; Amundson, R.; Bol, R.; Collins, C.; Lal, R.; Leifeld, J.; et al. Towards a global-scale soil climate mitigation strategy. Nat. Commun. 2020, 11, 5427. [CrossRef] [PubMed]

25. Galang, M.A.; Markewitz, D.; Morris, L.A.; Bussell, P. Land use change and gully erosion in the Piedmont region of South Carolina. J. Soil Water Conserv. 2007, 62, 122-129.

26. Kroeger, T.; Casey, F. An assessment of market-based approaches to providing ecosystem services on agricultural lands. Ecol. Econ. 2007, 321-332. [CrossRef]

27. Greainer, L.; Keller, A.; Grêt-Regamey, A.; Papritz, A. Soil function assessment: Review of methods for quantifying the contributions of soils to ecosystem services. Land Use Policy 2017, 69, 224-237. [CrossRef] 